\title{
Time-Consistent Portfolio Selection under Short-Selling Prohibition: From Discrete to Continuous Setting*
}

\author{
A. Bensoussan ${ }^{\dagger}$, K. C. Wong ${ }^{\ddagger}$, S. C. P. Yam ${ }^{\S}$, and S. P. Yung ${ }^{\ddagger}$
}

\begin{abstract}
In this paper, we study the time consistent strategies in the mean-variance portfolio selection with short-selling prohibition in both discrete and continuous time settings. Recently, [T. Björk, A. Murgoci, and X. Y. Zhou, Math. Finance, 24 (2014), pp. 1-24] considered the problem with state dependent risk aversion in the sense that the risk aversion is inversely proportional to the current wealth, and they showed that the time consistent control is linear in wealth. Considering the counterpart of their continuous time equilibrium control in the discrete time framework, the corresponding "optimal" wealth process can take negative values; and this negativity in wealth will lead the investor to a risk seeker which results in an unbounded value function that is economically unsound; even more, the limiting of the discrete solutions has shown to be their obtained continuous solution in [T. Björk, A. Murgoci, and X. Y. Zhou, Math. Finance, 24 (2014), pp. 1-24]. To deal this limitation, we eliminate the chance of getting nonpositive wealth by prohibiting short-selling. Using backward induction, the equilibrium control in discrete time setting is shown to be linear in wealth. An application of the extended Hamilton-Jacobi-Bellman equation (see [T. Björk and A. Murgoci, A General Theory of Markovian Time Inconsistent Stochastic Control Problems, working paper, Stockholm School of Economics, Stockholm, Sweden, 2010]) makes us also conclude that the continuous time equilibrium control is also linear in wealth with investment to wealth ratio satisfying an integral equation uniquely. We also show that the discrete time equilibrium controls converge to that in continuous time setting. Finally, in numerical studies, we illustrate that the constrained strategy in continuous setting can outperform the unconstrained one in some situations as depicted in Figure 8.
\end{abstract}

Key words. portfolio management, equilibrium Markovian control, wealth dependent risk aversion, extended Hamilton-Jacobi-Bellman, short-selling prohibition

AMS subject classifications. $60 \mathrm{H} 20,91 \mathrm{~A} 10,91 \mathrm{~B} 70,91 \mathrm{G} 10$

DOI. $10.1137 / 130914139$

${ }^{*}$ Received by the editors March 22, 2013; accepted for publication (in revised form) November 18, 2013; published electronically March 6, 2014.

http://www.siam.org/journals/sifin/5/91413.html

${ }^{\dagger}$ International Center for Decision and Risk Analysis, Naveen Jindal School of Management, The University of Texas at Dallas, Richardson, TX 75080, Graduate Department of Financial Engineering, Ajou University, Suwon, South Korea, and Department of Systems Engineering and Engineering Management, City University of Hong Kong, Hong Kong (axb046100@utdallas.edu). This author was supported by the WCU (World Class University) program through the National Research Foundation of Korea funded by the Ministry of Education, Science and Technology (R31-20007), the National Science Foundation under grant DMS-1303775, and the Research Grant Council of Hong Kong Special Administrative Region under grant GRF 500113.

${ }^{\ddagger}$ Department of Mathematics, The University of Hong Kong, Hong Kong (wongkc89@hku.hk, spyung@hku.hk). The second author was supported by The University of Hong Kong, and the present work constitutes a part of his work for his postgraduate dissertation. The fourth author was supported by an HKU internal grant of code 201109176016.

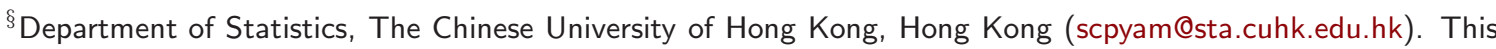
author was supported by the Hong Kong RGC GRF 404012 with the project title, "Advanced Topics in Multivariate Risk Management in Finance and Insurance," Chinese University of Hong Kong Direct grant 2010/2011 Project ID: 2060422, and Chinese University of Hong Kong Direct grant 2011/2012 Project ID: 2060444. 
1. Introduction. Since the first introduction in [18], the mean-variance portfolio selection has become a key research topic, in which the investor aims to determine the optimal portfolio that minimizes the variance risk of the terminal wealth subject to a predetermined budget constraint and at an arbitrary terminal expected return level; [19] and [22] later extended his model to multiperiod and continuous time settings, respectively, by using tools from stochastic control theory.

Due to the nonlinear expectation and dependence of current state in the mean-variance utility (see [3]), the usual tower property fails to hold, and the corresponding optimal portfolio selection problem is time inconsistent in the sense that it does not admit the Bellman optimality principle; that is, an optimal control that optimizes the mean-variance utility at time zero need not be optimal at a later time; as an example, see the optimal control in [5] and [6], which is one depending on both the current and the initial states.

Time inconsistency in optimization problems was first studied in [26]. There are basically three different approaches to providing solution concept for these time-inconsistent problems. First, under the notion of precommitment, only the solution that optimizes the initial objective function would be considered, whether it is optimal for the objective function at a later time or not is not relevant; relevant literature includes [5], [6], and [17]. Second, an agent primarily adopts the strategy that optimizes the objective function on the first day, and then on the next day, he will give up this strategy and use a new one that optimizes the objective function on the second day, and so on.

The third approach originated in [21] and [26], where the primitive idea of time-consistent strategies for time-inconsistent problems was provided; however, [20] later pointed out the nonexistence of such strategies in certain cases. In regard to this consideration, [20] treated time-inconsistent problems as a noncooperative game, in which strategies at different time points are planned by different players who aim to optimize their own objective functions; Nash equilibrium of these strategies was then utilized to define the time-consistent strategy for the agent of the original problem. This game theoretic approach and its extensions could be found in some recent works with an application in solving for the time-inconsistent consumption problems with nonclassical discounting utility, such as [3], [8], [9], and [10]. Specifically, [10] provided a precise definition of the (time-consistent) equilibrium control in continuous time settings, such that the control will be still an equilibrium one for any subproblems over an arbitrary confined time interval before the planning horizon.

Following their idea from [10], [3] studied the time-inconsistent control problem in a general Markovian framework in both discrete time and continuous time settings, and derived the extended Hamilton-Jacobi-Bellman (HJB) equation together with the verification theorem, which gives the necessary and sufficient condition of "equilibrium controls". Apart from the classical mean-variance utility model and the consumption and investment model with nonclassical (hyperbolic) discount, the time-inconsistent Markov framework in [3] has other further extensions and applications: collective objectives [16], recursive utility [25], and assetliability management [28].

In view of the complexity, calibrating an equilibrium control strategy is normally easier than computing a precommitment policy. To obtain the equilibrium strategy, we need only solve for the extended HJB; see, e.g., [3], [10], and [28]. On the other hand, to obtain the optimal control or precommitment solution, we have to deal with a couple of Fokker-

Copyright (c) by SIAM. Unauthorized reproduction of this article is prohibited. 
Planck and HJB equations (see [2] for detailed discussions), which commonly involves more computationally intensive considerations.

[1] and [3] studied the mean-variance portfolio selection and provided a time-consistent strategy via a game theoretic approach. It is important to note that their obtained common equilibrium control is completely independent of the current state; in spite of this independence, [4] reformulated the portfolio selection problem with wealth-dependent risk aversion. In particular, they illustrated that the equilibrium control is dependent on the current wealth if the risk aversion is inversely proportional to the current wealth. On the other hand, [28] adopted the asset and liability modeling in [6] with regime switching market parameters. [28] explicitly established that the equilibrium control is affine in current liability, while the equilibrium value function is affine in current surplus and is quadratic in current liability; that is, they both depend on the current state.

In this paper, we study the mean-variance portfolio selection with risk aversion being inversely proportional to the current wealth under short-selling prohibition first in the discrete time framework and then in its continuous time counterpart. In an earlier work, [4] also considered the similar time-inconsistent problem without short-selling prohibition. Their continuous model essentially relies on the positivity of the current wealth. Under no shortselling prohibition, there could be admissible controls leading to a nonpositive wealth process; nevertheless their obtained equilibrium control is linear in wealth, which in turn guarantees positivity of the optimal wealth process at any time almost surely. Even more, if one considers the discrete analogue of their continuous model, it is quite obvious to see the possibility that the wealth process can take nonpositive values even with the control being linear in wealth (see Remark 2.6 in section 2). In the present setting, the negative wealth will definitely lead to negative risk aversion, and now instead of risk averse, the investor becomes a risk seeker, and hence the corresponding mean-variance utility could be unbounded! This observation makes apparent the economic limitation of their continuous counterpart in the discrete framework. To avoid the possibility of taking nonpositive values for the wealth process, we forbid short-selling allowance on both stocks and riskless bonds in our work and aim at establishing the corresponding equilibrium controls for the mean variance portfolio with wealth-dependent risk aversion in both discrete and continuous time settings.

Considering constrained optimal controls is a very interesting and highly nontrivial problem in stochastic control theory. For example, in portfolio selection, in practice it is not ethical to allow an unlimited amount of short selling, and hence some definite measures such as this one imposes on the admissible control. Along this direction, there are a number of studies in the literature; for example, [23] and [24] studied the consumption and investment problem when selling stocks short is prohibited (but borrowing the riskless asset is allowed). [7] generalized the portfolio constraints to a closed, convex, nonempty subset; and later, [27] applied their result to study the problem under money borrowing constraints and stock shortsale restrictions. On the other hand, [13] studied the mean-variance portfolio selection with the portfolio taking values in a predetermined constrained set via the precommitment approach. In order to tackle these portfolio selection problems under constraints, all the above mentioned authors converted the primal problem into an unconstrained dual optimization problem. In contrast, [15] used the precommitment solution to the mean-variance problem with stock short-selling prohibition in terms of the solution of two extended stochastic Riccati

Copyright (c) by SIAM. Unauthorized reproduction of this article is prohibited. 
equations. Rather than constraining the portfolio, [12] studied the investment and consumption problem with no bankruptcy, i.e., negative wealth prohibition. To our knowledge, there is still very limited theoretical study via the time-consistent approach on the time-inconsistent control problem with constraints; though [11] studied the mean-variance problem via the time-consistent approach with constraints in investment policy, they provided only a numerical study on determining the time-consistent solution but not in any analytic form.

In section 2 , we review the unconstrained mean-variance portfolio selection in both the continuous time and discrete time frameworks via the time-consistent approach; also in this section, the economic limitation of the corresponding discrete counterpart of the continuous model (as studied by [4]) without short-selling constraints becomes apparent. In section 3, we introduce the formulation of the discrete time mean-variance portfolio selection problem. In section 4, we solve for the discrete time problem by using backward recursion and establish the equilibrium control in Theorem 4.1. In section 5, we introduce the formulation of the continuous time mean-variance problem and state the corresponding extended HJB equations for the mean-variance problem in Theorem 5.1. In section 6, we make use of a suitable ansatz in solving for the extended HJB system obtained in Theorem 5.1, and then we prove the existence and the uniqueness of the solution in Theorems 6.1 and 6.2. Furthermore, by utilizing the results in section 3 , we also show that the equilibrium controls of a sequence of canonical discrete time models as stated in Theorem 6.4 will converge to the equilibrium control of the continuous time model as introduced in section 5. Numerical simulation will be provided by comparing the performance of different constraints in control in section 7 , and we conclude in section 8. The main technical details in Theorems 5.1 and 6.4 are given in the appendices.

2. Unconstrained time-consistent optimization. In the mean-variance portfolio selection problem with wealth dependent risk aversion, we seek an optimal control $u$ to maximize the following objective functional:

$$
J(t, x ; u):=\mathbb{E}_{t, x}\left[X_{T}^{u}\right]-\frac{\gamma}{2 x} \operatorname{Var}_{t, x}\left[X_{T}^{u}\right],
$$

in which the wealth dynamic $d X_{t}^{u}=\left(r X_{t}^{u}+\alpha u_{t}\right) d t+\sigma u_{t} d W_{t}$, where $\gamma$ is a risk-aversion coefficient, $r$ is the riskless return rate, $\alpha:=\mu-r$ represents the net appreciation rate of the risky asset with $\mu$ being the appreciation rate of the risky asset, $\sigma$ is the volatility rate of the risky asset, and finally $W_{t}$ represents Brownian motion. For the sake of simplifying notation, we denote that $\mathbb{E}_{t, x}\left[X_{T}^{u}\right]=\mathbb{E}\left[X_{T}^{u} \mid X_{t}=x\right]$ and $\operatorname{Var}_{t, x}\left[X_{T}^{u}\right]=\operatorname{Var}\left[X_{T}^{u} \mid X_{t}=x\right]$. The class of all admissible controls, $\mathcal{U}^{c}$, is the collection of all real-valued Markovian controls.

Note that the objective functional $J$ is nonlinear in $\mathbb{E}_{t, x}\left[X_{T}^{u}\right]$, which cannot be linearly separated from initial state in $J$, and so the corresponding optimization problem is time inconsistent in the sense that the Bellman optimality principle does not hold (see [3]); that is, though any obtained optimal control can optimize $J$ at time 0 , the same control cannot guarantee the optimality of $J$ at all future times.

To deal with similar time-inconsistent optimization problems, it is getting popular in the recent literature to formulate the problem as a noncooperative game (with each time point as a player) and then look for a certain control in the equilibrium sense: in [3] and [10], the following definition of equilibrium control is provided in the class of all Markovian controls

Copyright (c) by SIAM. Unauthorized reproduction of this article is prohibited. 
for the time-inconsistent control problem in the continuous time setting as follows; a similar definition of equilibrium control in the discrete setting will be given in Definition 2.3.

Definition 2.1. A Markovian control $\hat{u}$ is said to be an equilibrium control if for every $u \in \mathcal{U}^{c}$,

$$
\liminf _{h \downarrow 0^{+}} \frac{J(t, x ; \hat{u})-J\left(t, x ; u_{h}\right)}{h} \geq 0 \quad \text { for any }(t, x) \in[0, T] \times \mathbb{R},
$$

where $u_{h}$ is given by

$$
u_{h}(s, y)=\left\{\begin{array}{lll}
u(s, y) & \text { for } & t \leq s<t+h \\
\hat{u}(s, y) & \text { for } & t+h \leq s \leq T
\end{array}\right.
$$

By solving for the HJB system in accordance with Definition 2.1 in [4], they obtain the following.

Theorem 2.2. The Markovian control $\hat{u}(t, x)=c(t) x$ is an equilibrium one for (2.1), where $c(t) \in C[0, T]$ uniquely solves for the integral equation:

$$
c(t)=\frac{\alpha}{\gamma \sigma^{2}}\left\{e^{-\int_{t}^{T}\left[r+\alpha c(s)+\sigma^{2} c^{2}(s)\right] d s}+\gamma e^{-\int_{t}^{T} \sigma^{2} c^{2}(s) d s}-\gamma\right\} .
$$

Note that this equilibrium solution is linear in the current wealth $x$.

Define $t_{n}:=n \epsilon$, where $\epsilon:=\frac{T}{N}$. Next, we consider the discrete time analogue of the portfolio selection problem with wealth dependent risk aversion in (2.1). The objective functional is defined as

$$
J(n, x ; u):=\mathbb{E}_{n, x}\left[X_{N}^{u}\right]-\frac{\gamma}{2 x} \operatorname{Var}_{n, x}\left[X_{N}^{u}\right] .
$$

Considering the continuous time dynamics of bond price $B_{t}$ and stock price $S_{t}$, by the direct application of Itô's formula, the changes of bond price and stock price over a step are given, respectively, by

$$
\begin{aligned}
B_{t_{n+1}} & =e^{r \epsilon} B_{t_{n}}, \\
S_{t_{n+1}} & =e^{\left(\mu-\frac{1}{2} \sigma^{2}\right) \epsilon+\sigma\left(W_{t_{n+1}}-W_{t_{n}}\right)} S_{t_{n}} .
\end{aligned}
$$

Therefore, the dynamic of the wealth at time $t_{n}, X_{n}$, is governed by

$$
X_{n+1}=\left(X_{n}-u_{n}\right) e^{r \epsilon}+u_{n} e^{\left(\mu-\frac{1}{2} \sigma^{2}\right) \epsilon+\sigma\left(W_{t_{n+1}}-W_{t_{n}}\right)},
$$

in which $u_{n}$ represents the control at time $t_{n}$. Again, we denote that $\mathbb{E}_{n, x}\left[X_{N}^{u}\right]=\mathbb{E}\left[X_{N}^{u} \mid X_{n}=\right.$ $x]$ and $\operatorname{Var}_{n, x}\left[X_{N}^{u}\right]=\operatorname{Var}\left[X_{N}^{u} \mid X_{n}=x\right]$. The set of all admissible controls, $\mathcal{U}^{d}$, is the collection of all real-valued Markovian controls. As before, this optimization problem is time inconsistent, which motivates us to look for the equilibrium (time-consistent; see [3] and [20]) control as defined next.

Definition 2.3. A Markovian control $\hat{u}$ is said to be an equilibrium control if for every $u \in \mathcal{U}^{d}$,

$$
J(n, x ; \hat{u}) \geq J(n, x ; \bar{u}) \quad \text { for any } x \in \mathbb{R}^{+}, n \in \mathcal{T},
$$

Copyright $\odot$ by SIAM. Unauthorized reproduction of this article is prohibited. 
where $\bar{u}$ is given by

$$
\bar{u}_{k}= \begin{cases}u_{k} & \text { if } k=n, \\ \hat{u}_{k} & \text { if } k>n .\end{cases}
$$

For further motivations of Definitions 2.1 and 2.3, and for the game theoretic approach on tackling some other time-inconsistent problems, one can consult [3], [10], and [20].

For any $x>0$, the optimization problem (2.3) admits a unique equilibrium control:

Theorem 2.4. The equilibrium control $\hat{u}$ is given by $\hat{u}_{n}(x)=C_{n} x$, where

$$
C_{n}:=\frac{A_{n+1}-\gamma e^{r \epsilon}\left(B_{n+1}-A_{n+1}^{2}\right)}{e^{2 r \epsilon}\left(e^{\alpha \epsilon}-1\right)^{2}\left(B_{n+1}-A_{n+1}^{2}\right)+\left(e^{\sigma^{2} \epsilon}-1\right) e^{2 \mu \epsilon} B_{n+1}} \frac{e^{r \epsilon}\left(e^{\alpha \epsilon}-1\right)}{\gamma},
$$

and $A_{n}$ and $B_{n}$ can be obtained recursively by

$$
\begin{aligned}
& A_{n}=\left(e^{r \epsilon}+e^{r \epsilon}\left(e^{\alpha \epsilon}-1\right) C_{n}\right) A_{n+1}, \quad A_{N}=1 ; \\
& B_{n}=\left[\left(e^{r \epsilon}+\left(e^{\alpha \epsilon}-1\right) C_{n}\right)^{2}+\left(e^{\sigma^{2} \epsilon}-1\right) e^{2 \mu \epsilon} C_{n}^{2}\right] B_{n+1}, \quad B_{N}=1 .
\end{aligned}
$$

A proof of Theorem 2.4 is obtained by solving for $\hat{u}$ by backward recursion; the approach is similar to that of Theorem 4.1 below, and the details are omitted. Furthermore, the solution in this discrete time model will converge to that in the continuous time setting as $N$ goes to infinity:

Theorem 2.5. If $c(t)$ is the solution of integral equation in Theorem 2.2 and $C_{n}$ are given in Theorem 2.4, then there exists $K>0$ independent of both $n$ and $N$ such that $\left|C_{n}-c\left(t_{n}\right)\right|<K \epsilon$.

Again we skip the details of the proof since it is almost the same as that of Theorem 6.4 below.

Remark 2.6. The solution in this discrete time framework relies on the assumption that $x>0$. Since the equilibrium control can take any real values, it is plausible that the ratio of the risky investment to wealth under the adoption of the equilibrium control, $C_{t_{n}}$, lies outside $[0,1]$, i.e., short-selling happens. In this scenario, even though the equilibrium control is linear in wealth, we cannot eliminate the possibility that in the next period the wealth takes nonpositive value:

$$
X_{n+1}=X_{n}\left[\left(1-C_{n}\right) e^{r \epsilon}+C_{n} e^{\left(\mu-\frac{1}{2} \sigma^{2}\right) \epsilon+\sigma\left(W_{t_{n+1}}-W_{t_{n}}\right)}\right] .
$$

Suppose that $X_{n}>0$. (i) If $C_{n}>1$ (short-selling riskless asset), $X_{n+1} \leq 0$ if $W_{t_{n+1}}-$ $W_{t_{n}}<\frac{\epsilon}{\sigma}\left[r-\left(\mu-\frac{1}{2} \sigma^{2}\right)\right]+\ln \left(\frac{C_{n}-1}{C_{n}}\right)$. (ii) If $C_{n}<0$ (short-selling risky asset), $X_{n+1} \leq 0$ if $W_{t_{n+1}}-W_{t_{n}}<\frac{\epsilon}{\sigma}\left[r-\left(\mu-\frac{1}{2} \sigma^{2}\right)\right]+\ln \left(\frac{1-C_{n}}{-C_{n}}\right)$.

In the continuous time framework, the equilibrium control of an unconstrained problem is linear in wealth, and now the wealth process becomes a geometric Brownian motion which avoids the possibility of getting negative. In contrast, the negative wealth may happen in the discrete time framework; however, its solution converges to that in the continuous time framework.

The negative wealth will then reinforce the investor to become a risk seeker, which seems to be counterintuitive. Furthermore, negative wealth also makes the wealth dependent risk aversion $\frac{\gamma}{x}$ economically unsound; namely, the investor could be very risk averse when he 
has $\$ 0.1$ in his pocket, while the investor becomes a strong risk seeker when he owes $\$ 0.1$. It sounds economically irrational that the investor behaves dramatically different just for this 0.2 dollar of difference! Third, mathematically, the optimal objective functional in $(2.3)$ will be unbounded under negative risk aversion. Thus, if we model the risk aversion to be inversely proportional to the wealth, we have to restrict plausible trading strategies that avoid bankruptcy.

It is usual to implement the portfolio management in a discrete time framework. The equilibrium strategy in an unconstrained discrete time framework can result in bankruptcy. It can also cause the loss of concavity of the mean-variance utility at some time, and thus its control cannot be implemented in a discrete time framework. Therefore, it is economically questionable that the unethical discrete time strategy will converge to the equilibrium control in [4] defined among all continuous time no bankruptcy strategies.

To overcome this anomaly, in the rest of this paper, to eliminate the chance of negative wealth, the admissible controls have to be constrained so that no short selling is allowed. We next consider the portfolio selection problem under short-selling prohibition in both discrete and continuous time frameworks.

3. Problem setting: Discrete time. Given the probability space $(\Omega, \mathcal{F}, \mathbb{P}), \mathbb{E}$ denotes the expectation with respect to real-life probability measure $\mathbb{P}$. The values of bonds and stocks are given by $n \in \mathcal{T}:=\{0,1, \ldots, N-1\}$,

$$
\begin{aligned}
B_{n+1} & =r_{n} B_{n}, \\
S_{n+1} & =R_{n} S_{n},
\end{aligned}
$$

where $r_{n}>0$ is the deterministic riskless return rate. $R_{n}$ is the positive random return rate of the stock, where $\mu_{n}:=\mathbb{E}\left[R_{n}\right]$ and $\sigma_{n}^{2}:=\operatorname{Var}\left[R_{n}\right]$. Also assume that $R_{i}$ and $R_{j}$ are independent for any $i \neq j$. Define $\mathcal{F}_{n}:=\sigma\left(R_{i}, i \leq n\right)$ and $\mathcal{F}=\cup_{n=0}^{N} \mathcal{F}_{n}$. Denote $u_{n}$ to be the amount of money invested on the stock at time $n$; the corresponding wealth process $X_{n}^{u}$ satisfies

$$
X_{n+1}^{u}=r_{n}\left(X_{n}^{u}-u_{n}\right)+R_{n} u_{n}
$$

Also we confine our control $u_{n}$ by

$$
p_{n} X_{n} \leq u_{n} \leq q_{n} X_{n},
$$

where $0 \leq p_{n} \leq q_{n} \leq 1$, subject to which short selling is not allowed, and the wealth has to be positive. Define the class of admissible controls $\mathcal{U}_{[p, q]}^{d}:=\left\{u_{n} \mid p_{n} X_{n} \leq u_{n} \leq q_{n} X_{n}, n \in \mathcal{T}\right\}$. The objective functional is defined as

$$
J(n, x ; u):=\mathbb{E}_{n, x}\left[X_{N}^{u}\right]-\frac{\gamma_{n}}{2 x} \operatorname{Var}_{n, x}\left[X_{N}^{u}\right],
$$

where $\mathbb{E}_{n, x}\left[X_{N}^{u}\right]=\mathbb{E}\left[X_{N}^{u} \mid X_{n}=x\right], \operatorname{Var}_{n, x}\left[X_{N}^{u}\right]=\operatorname{Var}\left[X_{N}^{u} \mid X_{n}=x\right]$, and the risk-aversion coefficient $\gamma_{n}>0$ for all $n \in \mathcal{T}$. From the dynamics of the wealth process, we have

$$
\begin{aligned}
\mathbb{E}_{n, x}\left[X_{n+1}^{u}\right] & =r_{n} x+\alpha_{n} u_{n}, \\
\mathbb{E}_{n, x}\left[\left(X_{n+1}^{u}\right)^{2}\right] & =\left(r_{n} x+\alpha_{n} u_{n}\right)^{2}+\sigma_{n}^{2} u_{n}^{2},
\end{aligned}
$$

Copyright $\odot$ by SIAM. Unauthorized reproduction of this article is prohibited. 
where $\alpha_{n}:=\mu_{n}-r_{n}$. We now look for the equilibrium solution as defined in Definition 2.3 for this $J(n, x ; u)$ in (3.3) under the dynamic (3.1) and constraint (3.2) (here we use $\mathcal{U}_{[p, q]}^{d}$ instead of $\left.\mathcal{U}^{d}\right)$.

Remark 3.1. The present problem is more general than the problem for (2.3) in the previous section except the additional constraint on all admissible controls. Besides, we here allow both the time varying of parameters and the risky return rate to be an arbitrary random variable. If one takes $r_{n}=e^{r \epsilon}$ and $R_{n}=e^{\left(\mu-\frac{1}{2} \sigma^{2}\right) \epsilon+\sigma\left(W_{t_{n+1}}-W_{t_{n}}\right)}$, the dynamics of the wealth process in two models coincide, and the independence of $R_{i}$ and $R_{j}$ for all $i \neq j$ follows from the stationary independent increments of Brownian motion. Moreover, for $p_{n}=0$ and $q_{n}=1$ for all $n$, constraint (3.2) is the standard short-selling prohibition.

4. Equilibrium solution for the discrete time problem. Define $f, g$ as

$$
\begin{aligned}
& f(n, x)=\mathbb{E}_{n, x}\left[\left(X_{N}^{\hat{u}}\right)^{2}\right], \\
& g(n, x)=\mathbb{E}_{n, x}\left[X_{N}^{\hat{u}}\right] .
\end{aligned}
$$

Theorem 4.1. The equilibrium control for the objective functional (3.3) subject to the $d y$ namics (3.1) and constraint (3.2), $\hat{u}$, is given by

$$
\hat{u}_{n}(x)=C_{n} x
$$

where $C_{n}$ is defined as follows: for $n<N$,

$$
C_{n}:=G_{n}\left(\frac{A_{n+1}-\gamma_{n} r_{n}\left(B_{n+1}-A_{n+1}^{2}\right)}{\alpha_{n}^{2}\left(B_{n+1}-A_{n+1}^{2}\right)+\sigma_{n}^{2} B_{n+1}} \frac{\alpha_{n}}{\gamma_{n}}\right),
$$

and $A_{n}$ and $B_{n}$ can be obtained recursively as

$$
\begin{aligned}
A_{n} & =\left(r_{n}+\alpha_{n} C_{n}\right) A_{n+1}, \\
A_{N} & =1 ; \\
B_{n} & =\left[\left(r_{n}+\alpha_{n} C_{n}\right)^{2}+\sigma_{n}^{2} C_{n}^{2}\right] B_{n+1}, \\
B_{N} & =1 ;
\end{aligned}
$$

and $G_{n}$ is a layer function defined as the following:

$$
G_{n}(x):= \begin{cases}q_{n} & \text { if } x>q_{n} \\ x & \text { if } x \in\left[p_{n}, q_{n}\right] \\ p_{n} & \text { if } x<p_{n} .\end{cases}
$$

Also, we have

$$
\begin{aligned}
& f(n, x)=B_{n} x^{2}, \\
& g(n, x)=A_{n} x
\end{aligned}
$$

and

$$
\begin{aligned}
B_{n} & >0, \\
B_{n}-A_{n}^{2} & \geq 0
\end{aligned}
$$

Copyright $\odot$ by SIAM. Unauthorized reproduction of this article is prohibited. 
for all $n \in \mathcal{T}$.

Note that if (4.8) and (4.9) hold, (4.10) and (4.11) can be interpreted in this way: $B_{n}$ represents the coefficients of $x^{2}$ for the conditional second moment of the terminal wealth up to time $n$, while $B_{n}-A_{n}^{2}$ represents the coefficients of $x^{2}$ for the conditional variance of the terminal wealth up to time $n$. And hence both of them should be nonnegative.

Proof of Theorem 4.1. Our proof relies on backward induction. It is trivial that (4.8)(4.11) hold for $n=N$. Assume that (4.3), (4.8)-(4.11) hold for $n \geq k+1$, and we now turn to establishing their validity for $n=k$. Let $u=\left(u_{k}, \hat{u}_{k+1}, \ldots, \hat{u}_{N}\right)$; by using the notion of $(4.1)$ and (4.2) and the tower property,

$$
\begin{aligned}
J(k, x ; u) & =\mathbb{E}_{k, x}\left[X_{N}^{u}\right]-\frac{\gamma_{k}}{2 x} \operatorname{Var}_{k, x}\left[X_{N}^{u}\right] \\
& =\mathbb{E}_{k, x}\left[\mathbb{E}_{k+1, X_{k+1}^{u_{k}}}\left[X_{N}^{\hat{u}}\right]\right]-\frac{\gamma_{k}}{2 x}\left(\mathbb{E}_{k, x}\left[\mathbb{E}_{k+1, X_{k+1}^{u_{k}}}\left[\left(X_{N}^{\hat{u}}\right)^{2}\right]\right]-\left(\mathbb{E}_{k, x}\left[\mathbb{E}_{k+1, X_{k+1}^{u_{k}}}\left[X_{N}^{\hat{u}}\right]\right]\right)^{2}\right) \\
& =\mathbb{E}_{k, x}\left[g\left(k+1, X_{k+1}^{u_{k}}\right)\right]-\frac{\gamma_{k}}{2 x}\left(\mathbb{E}_{k, x}\left[f\left(k+1, X_{k+1}^{u_{k}}\right)\right]-\left(\mathbb{E}_{k, x}\left[g\left(k+1, X_{k+1}^{u_{k}}\right)\right]\right)^{2}\right) .
\end{aligned}
$$

Since (4.8) and (4.9) hold for $n=k+1$, we have

$$
\begin{aligned}
J(k, x ; u)= & A_{k+1} \mathbb{E}_{k, x}\left[X_{k+1}^{u_{k}}\right]-\frac{\gamma_{k}}{2 x}\left(B_{k+1} \mathbb{E}_{k, x}\left[\left(X_{k+1}^{u_{k}}\right)^{2}\right]-A_{k+1}^{2}\left(\mathbb{E}_{k, x}\left[X_{k+1}^{u_{k}}\right]\right)^{2}\right) \\
= & A_{k+1}\left(r_{k} x+\alpha_{k} u_{k}\right)-\frac{\gamma_{k}}{2 x}\left(B_{k+1}\left[\left(r_{k} x+\alpha_{k} u_{k}\right)^{2}+\sigma_{k}^{2} u_{k}^{2}\right]-A_{k+1}^{2}\left(r_{k} x+\alpha_{k} u_{k}\right)^{2}\right) \\
= & -\frac{\gamma_{k}}{2 x}\left(\alpha_{k}^{2}\left(B_{k+1}-A_{k+1}^{2}\right)+\sigma_{k}^{2} B_{k+1}\right) u_{k}^{2}+\alpha_{k}\left(A_{k+1}-\gamma_{k} r_{k}\left(B_{k+1}-A_{k+1}^{2}\right)\right) u_{k} \\
& +r_{k} x\left(A_{k+1}-\frac{\gamma_{k}}{2} r_{k}\left(B_{k+1}-A_{k+1}^{2}\right)\right) .
\end{aligned}
$$

The equilibrium control at $k$ can be obtained by maximizing the above quadratic function of $u_{k}$. From the hypothesis that (4.10) and (4.11) hold for $n=k+1$, this quadratic function is strictly concave in $u_{k}$. By the first order condition, the quadratic function is maximized by adopting (4.3); so (4.3) holds for $n=k$. Furthermore,

$$
\begin{aligned}
f(k, x) & =\mathbb{E}_{k, x}\left[\left(X_{N}^{\hat{u}}\right)^{2}\right] \\
& =\mathbb{E}_{k, x}\left[f\left(k+1, X_{k+1}^{\hat{u}}\right)\right] \\
& =B_{k+1} \mathbb{E}_{k, x}\left[\left(X_{k+1}^{\hat{u}}\right)^{2}\right] \\
& =B_{k+1}\left[\left(r_{k} x+\alpha_{k} C_{k} x\right)^{2}+\sigma_{k}^{2}\left(C_{k} x\right)^{2}\right] \\
& =B_{k+1}\left[\left(r_{k}+\alpha_{k} C_{k}\right)^{2}+\sigma_{k}^{2} C_{k}^{2}\right] x^{2}, \\
g(k, x) & =\mathbb{E}_{k, x}\left[X_{N}^{\hat{u}}\right] \\
& =\mathbb{E}_{k, x}\left[g\left(k+1, X_{k+1}^{\hat{u}}\right)\right] \\
& =A_{k+1} \mathbb{E}_{k, x}\left[X_{k+1}^{\hat{u}}\right] \\
& =A_{k+1}\left(r_{k}+\alpha_{k} C_{k}\right) x .
\end{aligned}
$$

Hence, (4.8) and (4.9) hold for $n=k$. Obviously, $B_{k+1}>0$ and $B_{k+1}-A_{k+1}^{2} \geq 0$ imply

$$
\begin{aligned}
B_{k} & =B_{k+1}\left[\left(r_{k}+\alpha_{k} C_{k}\right)^{2}+\sigma_{k}^{2} C_{k}^{2}\right]>0 ; \\
B_{k}-A_{k}^{2} & =\left(r_{k}+\alpha_{k} C_{k}\right)^{2}\left(B_{k+1}-A_{k+1}^{2}\right)+\sigma_{k}^{2} C_{k}^{2} B_{k+1} \geq 0 .
\end{aligned}
$$

Therefore, (4.10) and (4.11) hold for $n=k$, and the claim follows by backward induction.

Copyright (C) by SIAM. Unauthorized reproduction of this article is prohibited. 
5. Problem setting: Continuous time model motivated by the discrete framework. Given the probability space $(\Omega, \mathcal{F}, \mathbb{P}), \mathbb{E}$ denotes the expectation with respect to $\mathbb{P}$, and $W_{t}$ denotes the standard $\mathbb{P}$-Brownian motion. The values of bonds and stocks are given by

$$
\begin{aligned}
d B_{t} & =r_{t} B_{t} d t, \\
d S_{t} & =\mu_{t} S_{t} d t+\sigma_{t} S_{t} d W_{t},
\end{aligned}
$$

where $r_{t}$ is the riskless return rate, and $\mu_{t}$ and $\sigma_{t}$ are the appreciation and the volatility rates of the stock, respectively. Also assume that $r_{t}, \mu_{t}$, and $\sigma_{t}$ are locally Lipschitz continuous and $\sigma_{t}^{2}>0$ on $[0, T]$. The dynamic of controlled wealth process is

$$
d X_{t}^{u}=\left(r_{t} X_{t}^{u}+\alpha_{t} u_{t}\right) d t+\sigma_{t} u_{t} d W_{t}
$$

where $\alpha_{t}:=\mu_{t}-r_{t}$. The Markovian admissible controls are confined by the constraint

$$
p_{t} X_{t} \leq u_{t} \leq q_{t} X_{t},
$$

where $p_{t}$ and $q_{t}$ are locally Lipschitz continuous functions. Define the class of all admissible controls $\mathcal{U}_{[p, q]}^{c}:=\left\{u_{t} \mid p_{t} X_{t} \leq u_{t} \leq q_{t} X_{t}, t \in[0, T]\right\}$. The objective functional is

$$
J(t, x ; u):=\mathbb{E}_{t, x}\left[X_{T}^{u}\right]-\frac{\gamma_{t}}{2 x} \operatorname{Var}_{t, x}\left[X_{T}^{u}\right],
$$

where $T<\infty$ and risk-aversion coefficient $\gamma_{t}$ is assumed to be positive and differentiable with bounded derivative. The definition of equilibrium control in the continuous time setting is given in Definition 2.1 (here we use $\mathcal{U}_{[p, q]}^{c}$ instead of $\mathcal{U}^{c}$ ). We can define the equilibrium value function by equilibrium control:

$$
V(t, x)=J(t, x ; \hat{u}) .
$$

By the extended HJB system and its corresponding verification theorem derived in [3], we have the following extended HJB system and the verification theorem for objective function $J(t, x ; u)$ in (5.3) subject to (5.1) and (5.2).

Theorem 5.1 (verification theorem). Suppose that there are functions $V, g, \hat{u}:[0, T] \times \mathbb{R}^{+} \rightarrow$ $\mathbb{R}, f:[0, T] \times \mathbb{R}^{+} \times[0, T] \times \mathbb{R}^{+} \rightarrow \mathbb{R}$ such that they satisfy the following system of equations:

$$
\begin{aligned}
V_{t}(t, x)-f_{s}(t, x, t, x)-\frac{\gamma_{t}^{\prime}}{2 x} g^{2}(t, x) & \\
+\sup _{u \in\left[p_{t} x, q_{t} x\right]}\left\{\left(r_{t} x+\alpha_{t} u\right)\left(V_{x}(t, x)-f_{y}(t, x, t, x)+\frac{\gamma_{t}}{2 x^{2}} g^{2}(t, x)\right)\right. & \\
+\frac{1}{2} \sigma_{t}^{2} u^{2}\left[V_{x x}(t, x)-f_{y y}(t, x, t, x)-2 f_{x y}(t, x, t, x)-\frac{\gamma_{t}}{x^{3}} g^{2}(t, x)\right. & \\
\left.\left.+2 \frac{\gamma_{t}}{x^{2}} g(t, x) g_{x}(t, x)-\frac{\gamma_{t}}{x} g_{x}^{2}(t, x)\right]\right\} & =0, \\
f_{t}(t, x, s, y)+\left(r_{t} x+\alpha_{t} \hat{u}\right) f_{x}(t, x, s, y)+\frac{1}{2} \sigma_{t}^{2} \hat{u}^{2} f_{x x}(t, x, s, y) & =0, \\
g_{t}(t, x)+\left(r_{t} x+\alpha_{t} \hat{u}\right) g_{x}(t, x)+\frac{1}{2} \sigma_{t}^{2} \hat{u}^{2} g_{x x}(t, x) & =0, \\
V(T, x) & =x, \\
f(T, x, s, y) & =x-\frac{\gamma_{s}}{2 y} x^{2}, \\
g(T, x) & =x,
\end{aligned}
$$

Copyright (C) by SIAM. Unauthorized reproduction of this article is prohibited. 
where the supremum in the first equation is attained at $\hat{u}(t, x)$ for all $x \in \mathbb{R}^{+}$.

Then $\hat{u}$ is an equilibrium control law for $J(t, x ; u)$ in (5.3) subject to (5.1) and (5.2), and $V$ is the corresponding equilibrium value function. Moreover, $f$ and $g$ have the following probabilistic representations:

$$
\begin{aligned}
f(t, x, s, y) & =\mathbb{E}_{t, x}\left[X_{T}^{\hat{u}}-\frac{\gamma_{s}}{2 y}\left(X_{T}^{\hat{u}}\right)^{2}\right] \\
g(t, x) & =\mathbb{E}_{t, x}\left[X_{T}^{\hat{u}}\right] .
\end{aligned}
$$

The proof of the above theorem is given in Appendix A.

Remark 5.2. The problem setting in this section allows a time varying parameter. It is more general than (2.1) except the constraint on the control. If we take $p_{t}=0$ and $q_{t}=1$ for all $t \in[0, T]$, constraint (5.2) is a standard short-selling prohibition.

6. Equilibrium control in continuous time problem. By considering the equilibrium control in the discrete time setting, we apply the following ansatz to the equilibrium control in the continuous time problem:

$$
\hat{u}=c(t) x .
$$

By the probabilistic representation in (5.10), we consider the following expression for $f$ and $g$ :

$$
\begin{aligned}
f(t, x, s, y) & =e^{\int_{t}^{T}\left[r_{\tau}+\alpha_{\tau} c(\tau)\right] d \tau} x-\frac{\gamma_{s}}{2 y} e^{2 \int_{t}^{T}\left[r_{\tau}+\alpha_{\tau} c(\tau)+\frac{1}{2} \sigma_{\tau}^{2} c^{2}(\tau)\right] d \tau} x^{2}, \\
g(t, x) & =e^{\int_{t}^{T}\left[r_{\tau}+\alpha_{\tau} c(\tau)\right] d \tau} x .
\end{aligned}
$$

By direct calculations, these $f$ and $g$ can be shown to satisfy (5.5) and (5.6). Furthermore, we also have the following expression for $V$ :

$$
V(t, x)=\left\{e^{\int_{t}^{T}\left[r_{s}+\alpha_{s} c(s)\right] d s}-\frac{\gamma_{t}}{2}\left[e^{2 \int_{t}^{T}\left[r_{s}+\alpha_{s} c(s)+\frac{1}{2} \sigma_{s}^{2} c^{2}(s)\right] d s}-e^{2 \int_{t}^{T}\left[r_{s}+\alpha_{s} c(s)\right] d s}\right]\right\} x .
$$

Standard derivation gives

$$
\begin{aligned}
& V_{x}(t, x)-f_{y}(t, x, t, x)+\frac{\gamma_{t}}{2 x^{2}} g^{2}(t, x) \\
= & e^{\int_{t}^{T}\left[r_{s}+\alpha_{s} c(s)\right] d s}-\gamma_{t}\left[e^{2 \int_{t}^{T}\left[r_{s}+\alpha_{s} c(s)+\frac{1}{2} \sigma_{s}^{2} c^{2}(s)\right] d s}-e^{2 \int_{t}^{T}\left[r_{s}+\alpha_{s} c(s)\right] d s}\right]
\end{aligned}
$$

and

$$
\begin{aligned}
& V_{x x}(t, x)-f_{y y}(t, x, t, x)-2 f_{x y}(t, x, t, x)-\frac{\gamma_{t}}{x^{3}} g^{2}(t, x)+2 \frac{\gamma_{t}}{x^{2}} g(t, x) g_{x}(t, x)-\frac{\gamma_{t}}{x} g_{x}^{2}(t, x) \\
= & -\frac{\gamma_{t}}{x} e^{2 \int_{t}^{T}\left[r_{s}+\alpha_{s} c(s)+\frac{1}{2} \sigma_{s}^{2} c^{2}(s)\right] d s} .
\end{aligned}
$$

Copyright $($ ) by SIAM. Unauthorized reproduction of this article is prohibited. 
Substituting these derivatives into (5.4), we have a concave quadratic equation in $u$ (where $V_{t}(t, x)$ is independent of $\left.u\right)$ :

$$
\begin{array}{r}
V_{t}(t, x)-f_{s}(t, x, t, x)-\frac{\gamma_{t}^{\prime}}{2 x} g^{2}(t, x) \\
+\sup _{u \in\left[p_{t}, q_{t}\right]}\left\{r _ { t } x \left(e^{\int_{t}^{T}\left[r_{s}+\alpha_{s} c(s)\right] d s}-\gamma_{t}\left[e^{2 \int_{t}^{T}\left[r_{s}+\alpha_{s} c(s)+\frac{1}{2} \sigma_{s}^{2} c^{2}(s)\right] d s}-e^{\left.2 \int_{t}^{T}\left[r_{s}+\alpha_{s} c(s)\right] d s\right]}\right)\right.\right. \\
+\alpha_{t}\left(e^{\int_{t}^{T}\left[r_{s}+\alpha_{s} c(s)\right] d s}-\gamma_{t}\left[e^{2 \int_{t}^{T}\left[r_{s}+\alpha_{s} c(s)+\frac{1}{2} \sigma_{s}^{2} c^{2}(s)\right] d s}-e^{2 \int_{t}^{T}\left[r_{s}+\alpha_{s} c(s)\right] d s}\right]\right) u \\
\left.-\frac{\gamma_{t}}{2 x} \sigma_{t}^{2} e^{2 \int_{t}^{T}\left[r_{s}+\alpha_{s} c(s)+\frac{1}{2} \sigma_{s}^{2} c^{2}(s)\right] d s} u^{2}\right\}=0 .
\end{array}
$$

The first order condition gives us with optimal $\hat{u}$

$$
\hat{u}(t, x)=G_{t}\left(\frac{\alpha_{t}}{\gamma_{t} \sigma_{t}^{2}}\left\{e^{-\int_{t}^{T}\left[r_{s}+\alpha_{s} c(s)+\sigma_{s}^{2} c^{2}(s)\right] d s}+\gamma_{t} e^{-\int_{t}^{T} \sigma_{s}^{2} c^{2}(s) d s}-\gamma_{t}\right\}\right) x
$$

where $G_{t}$ is a layer function defined as the following:

$$
G_{t}(x):= \begin{cases}q_{t} & \text { if } x>q_{t} \\ x & \text { if } x \in\left[p_{t}, q_{t}\right] \\ p_{t} & \text { if } x<p_{t} .\end{cases}
$$

Since $p_{t}$ and $q_{t}$ are Lipschitz continuous, the layer function $G_{t}$ is jointly Lipschitz:

$$
\left|G_{t}(x)-G_{s}(y)\right| \leq|x-y|+K|t-s|
$$

By comparing coefficients of $(6.4)$ and $\hat{u}=c(t) x$, we ought to have $c(t)$ to satisfy the integral equation

$$
c(t)=G_{t}\left(\frac{\alpha_{t}}{\gamma_{t} \sigma_{t}^{2}}\left\{e^{-\int_{t}^{T}\left[r_{s}+\alpha_{s} c(s)+\sigma_{s}^{2} c^{2}(s)\right] d s}+\gamma_{t} e^{-\int_{t}^{T} \sigma_{s}^{2} c^{2}(s) d s}-\gamma_{t}\right\}\right) .
$$

Theorem 6.1. Assume that $c$ is the unique solution in $C[0, T]$ of the integral equation (6.6). If $V, g:[0, T] \times \mathbb{R}^{+} \rightarrow \mathbb{R}, f:[0, T] \times \mathbb{R}^{+} \times[0, T] \times \mathbb{R}^{+} \rightarrow \mathbb{R}$ are given by (6.3), (6.2), and (6.1), respectively, and $\hat{u}:[0, T] \times \mathbb{R}^{+} \rightarrow \mathbb{R}$ is given by $\hat{u}(t, x)=c(t) x$, then $V, f, g, \hat{u}$ satisfy the HJB system given by (5.4)-(5.9). Moreover, by the verification theorem (Theorem 5.1), $\hat{u}$ is an equilibrium control law, $V$ is an equilibrium value function, and $f, g$ have probabilistic representation as in (5.10).

Proof. Equations (5.5)-(5.9) are obviously satisfied by the choices of $f, g$, and $\hat{u}$ as in (6.2) and (6.1). By (6.4) and the concavity, we have shown that the supremum of (5.4) is attained at $\hat{u}$. We only have to show (5.4); indeed, by the choices of $f, g$, and $V$ as in (6.2), (6.1), and (6.3), we have

$$
V(t, x)=f(t, x, t, x)+\frac{\gamma_{t}}{2 x}[g(t, x)]^{2},
$$

Copyright $\odot$ by SIAM. Unauthorized reproduction of this article is prohibited. 
and thus we can express the following derivatives of $V$ as

$$
\begin{aligned}
V_{t}(t, x)= & f_{t}(t, x, t, x)+f_{s}(t, x, t, x)+\frac{\gamma_{t}}{x} g_{t}(t, x) g(t, x)+\frac{\gamma_{t}^{\prime}}{2 x} g^{2}(t, x), \\
V_{x}(t, x)= & f_{x}(t, x, t, x)+f_{y}(t, x, t, x)-\frac{\gamma_{t}}{2 x^{2}} g^{2}(t, x)+\frac{\gamma}{x} g_{x}(t, x) g(t, x), \\
V_{x x}(t, x)= & f_{x x}(t, x, t, x)+2 f_{x y}(t, x, t, x)+f_{y y}(t, x, t, x)+\frac{\gamma_{t}}{x^{3}} g^{2}(t, x)-\frac{2 \gamma_{t}}{x^{2}} g_{x}(t, x) g(t, x) \\
& +\frac{\gamma_{t}}{x} g_{x x}(t, x) g(t, x)+\frac{\gamma_{t}}{x} g_{x}^{2}(t, x) .
\end{aligned}
$$

Replacing $u$ by $\hat{u}$, the (5.4) can be rewritten as

$$
\begin{aligned}
& \quad V_{t}(t, x)-f_{s}(t, x, t, x)-\frac{\gamma_{t}^{\prime}}{2 x} g^{2}(t, x)+\left\{\left(r_{t} x+\alpha_{t} \hat{u}\right)\left(V_{x}(t, x)-f_{y}(t, x, t, x)+\frac{\gamma_{t}}{2 x^{2}} g^{2}(t, x)\right)\right. \\
& +\frac{1}{2} \sigma_{t}^{2} \hat{u}^{2}\left[V_{x x}(t, x)-f_{y y}(t, x, t, x)-2 f_{x y}(t, x, t, x)-\frac{\gamma_{t}}{x^{3}} g^{2}(t, x)\right. \\
& \left.\left.\quad+2 \frac{\gamma_{t}}{x^{2}} g(t, x) g_{x}(t, x)-\frac{\gamma_{t}}{x} g_{x}^{2}(t, x)\right]\right\} \\
& =f_{t}(t, x, t, x)+\left(r_{t} x+\alpha_{t} \hat{u}\right) f_{x}(t, x, t, x)+\frac{1}{2} \sigma_{t}^{2} \hat{u}^{2} f_{x x}(t, x, t, x) \\
& \quad+\frac{\gamma_{t}}{x} g(t, x)\left[g_{t}(t, x)+\left(r_{t} x+\alpha_{t} \hat{u}\right) g_{x}(t, x)+\frac{1}{2} \sigma_{t}^{2} \hat{u}^{2} g_{x x}(t, x)\right] \\
& =0 .
\end{aligned}
$$

The last equality follows from (5.5) and (5.6).

What remains now is to verify whether the integral equation (6.6) admits a unique solution in $C[0, T]$.

Theorem 6.2. The integral equation (6.6) admits a unique solution $c \in C[0, T]$.

Remark 6.3. The existence proof of integral equation (6.6) in Theorem 6.2 and the integral equation in [4] are similar. We both construct an iteration scheme for the integral equation and establish the existence of a convergence subsequence in accordance with the Arzela-Ascoli theorem. On the other hand, in [4], it does not seem clear enough on showing the limit of the subsequence actually satisfying the integral equation; indeed, just a subsequential convergence does not suffice to take the limit on both sides of the corresponding recursive relation. In our proof of Theorem 6.2, we conclude that the limit of the subsequence is the limit of the sequence by showing that the iteration (6.7) is actually Cauchy. Nevertheless, we can similarly show that the corresponding iteration scheme in [4] is also Cauchy in spite of Theorem 4.9 in [4].

Proof of Theorem 6.2. In the following, for the sake of notation, $K^{\prime}$ represents different constants at different lines. The proof is based on an application of the Arzela-Ascoli theorem. Construct a sequence $c_{n} \in C[0, T]$ as follows:

$$
\begin{aligned}
c_{0}(t) & =G_{t}\left(\frac{\alpha_{t}}{\gamma_{t} \sigma_{t}^{2}}\right), \\
c_{n+1}(t) & =G_{t}\left(\frac{\alpha_{t}}{\gamma_{t} \sigma_{t}^{2}} d_{n}(t)\right),
\end{aligned}
$$

Copyright $\odot$ by SIAM. Unauthorized reproduction of this article is prohibited. 
where $d_{n}(t):=e^{-\int_{t}^{T}\left[r_{s}+\alpha_{s} c_{n}(s)+\sigma_{s}^{2} c_{n}^{2}(s)\right] d s}+\gamma_{t} e^{-\int_{t}^{T} \sigma_{s}^{2} c_{n}^{2}(s) d s}-\gamma_{t}$. Since $0 \leq p_{t} \leq G_{t} \leq q_{t} \leq 1$ for all $t \in[0, T], c_{n}$ is uniformly bounded by 0 and 1 in $[0, T]$. We next show that $\left\{c_{n}\right\}$ is equicontinuous. Note that all $r_{t}, \alpha_{t}, \sigma_{t}, \gamma_{t}$ are Lipschitz continuous (since $\gamma_{t}$ has bounded derivative), and so they are uniformly bounded on $[0, T]$. Define

$$
\begin{aligned}
r & :=\max _{t \in[0, T]}\left|r_{t}\right|, \alpha:=\max _{t \in[0, T]}\left|\alpha_{t}\right|, \bar{\sigma}:=\max _{t \in[0, T]}\left|\sigma_{t}\right|, \bar{\gamma}:=\max _{t \in[0, T]}\left|\gamma_{t}\right|<\infty, \\
\underline{\sigma} & :=\min _{t \in[0, T]} \sigma_{t}, \underline{\gamma}:=\min _{t \in[0, T]} \gamma_{t}>0 .
\end{aligned}
$$

For any $s, t \in[0, T]$, we have

$$
\begin{aligned}
& \left|c_{n+1}(t)-c_{n+1}(s)\right| \\
= & \left|G_{t}\left(\frac{\alpha_{t}}{\gamma_{t} \sigma_{t}^{2}} d_{n}(t)\right)-G_{s}\left(\frac{\alpha_{s}}{\gamma_{s} \sigma_{s}^{2}} d_{n}(s)\right)\right| \\
\leq & \left|\frac{\alpha_{t}}{\gamma_{t} \sigma_{t}^{2}} d_{n}(t)-\frac{\alpha_{s}}{\gamma_{s} \sigma_{s}^{2}} d_{n}(s)\right|+K|t-s| \\
\leq & \left|\frac{\alpha_{t}}{\sigma_{t}^{2} \gamma_{t}} d_{n}(t)-\frac{\alpha_{s}}{\sigma_{t}^{2} \gamma_{t}} d_{n}(t)\right|+\left|\frac{\alpha_{s}}{\sigma_{t}^{2} \gamma_{t}} d_{n}(t)-\frac{\alpha_{s}}{\sigma_{t}^{2} \gamma_{s}} d_{n}(t)\right|+\left|\frac{\alpha_{s}}{\sigma_{t}^{2} \gamma_{s}} d_{n}(t)-\frac{\alpha_{s}}{\sigma_{s}^{2} \gamma_{s}} d_{n}(t)\right| \\
& +\left|\frac{\alpha_{s}}{\sigma_{s}^{2} \gamma_{s}} d_{n}(t)-\frac{\alpha_{s}}{\sigma_{s}^{2} \gamma_{s}} d_{n}(s)\right|+K|t-s| \\
= & \left|\alpha_{t}-\alpha_{s}\right|\left|\frac{d_{n}(t)}{\gamma_{t} \sigma_{t}^{2}}\right|+\left|\gamma_{t}-\gamma_{s}\right|\left|\frac{\alpha_{s} d_{n}(t)}{\gamma_{t} \gamma_{s} \sigma_{t}^{2}}\right|+\left|\sigma_{t}-\sigma_{s}\right|\left|\frac{\alpha_{s} d_{n}(t)\left(\sigma_{t}+\sigma_{s}\right)}{\sigma_{t}^{2} \sigma_{s}^{2} \gamma_{s}}\right| \\
& +\left|d_{n}(t)-d_{n}(s)\right| \frac{\alpha_{s}}{\sigma_{s}^{2}}|+K| t-s \mid \\
\leq & K^{\prime}\left(\left|d_{n}(t)\right||t-s|+\left|d_{n}(t)-d_{n}(s)\right|+|t-s|\right) .
\end{aligned}
$$

The equicontinuity of $\left\{c_{n}\right\}$ follows if we can show that $d_{n}(t)$ is uniformly bounded and $d_{n}(t)$ is Lipschitz continuous:

$$
-\bar{\gamma} \leq d_{n}(t) \leq e^{-\int_{t}^{T}\left[r_{s}+\alpha_{s} c_{n}(s)+\sigma_{s}^{2} c_{n}^{2}(s)\right] d s}+\gamma_{t} e^{-\int_{t}^{T} \sigma_{s}^{2} c_{n}^{2}(s) d s} \leq e^{(r+\alpha) T}+\bar{\gamma} .
$$

Therefore, $d_{n}(t)$ is uniformly bounded. Furthermore,

$$
\begin{aligned}
& \left|d_{n}(t)-d_{n}(s)\right| \\
\leq & \left|e^{-\int_{t}^{T}\left[r_{u}+\alpha_{u} c_{n}(u)+\sigma_{u}^{2} c_{n}^{2}(u)\right] d u}-e^{-\int_{s}^{T}\left[r_{u}+\alpha_{u} c_{n}(u)+\sigma_{u}^{2} c_{n}^{2}(u)\right] d u}\right| \\
& +\left|\gamma_{t}-\gamma_{s}\right|\left|e^{-\int_{t}^{T} \sigma_{u}^{2} c_{n}^{2}(u) d u}-1\right|+\gamma_{s}\left|e^{-\int_{t}^{T} \sigma_{u}^{2} c_{n}^{2}(u) d u}-e^{-\int_{s}^{T} \sigma_{u}^{2} c_{n}^{2}(u) d u}\right| \\
\leq & e^{-\int_{t}^{T}\left[r_{u}+\alpha_{u} c_{n}(u)+\sigma_{u}^{2} c_{n}^{2}(u)\right] d u}\left|1-e^{-\int_{s}^{t}\left[r_{u}+\alpha_{u} c_{n}(u)+\sigma_{u}^{2} c_{n}^{2}(u)\right] d u}\right| \\
& +\left|\gamma_{t}-\gamma_{s}\right|+\gamma_{s} e^{-\int_{t}^{T} \sigma_{u}^{2} c_{n}^{2}(u) d u}\left|1-e^{-\int_{s}^{t} \sigma_{u}^{2} c_{n}^{2}(u) d u}\right|
\end{aligned}
$$

Copyright (C) by SIAM. Unauthorized reproduction of this article is prohibited. 


$$
\begin{aligned}
& \leq K^{\prime} e^{(r+\alpha) T}\left|\int_{s}^{t}\left[r_{u}+\alpha_{u} c_{n}(u)+\sigma_{u}^{2} c_{n}^{2}(u)\right] d u\right|+K^{\prime}|t-s|+K^{\prime} e^{\sigma^{2} T}\left|\int_{s}^{t} \sigma_{u}^{2} c_{n}^{2}(u) d u\right| \\
& \leq K^{\prime} e^{(r+\alpha) T}\left(r+\alpha+\bar{\sigma}^{2}\right)|t-s|+K^{\prime}|t-s|+K^{\prime} e^{\sigma^{2} T} \bar{\sigma}^{2}|t-s| \leq K^{\prime}|t-s|
\end{aligned}
$$

The Arzela-Ascoli theorem concludes with the existence of a subsequence $c_{n_{k}}$ and $c:[0, T] \rightarrow$ $\mathbb{R}$ such that $c_{n_{k}} \rightarrow c \in C[0, T]$ uniformly on $[0, T]$. Next, we shall show that for any $t \in[0, T]$, $\left\{c_{n}(t)\right\}_{n \in \mathbb{N}}$ is a Cauchy sequence, so $c_{n}(t) \rightarrow c(t)$ uniformly. Define $\bar{c}_{n}:=c_{n}-c_{n-1}$. By definition, $c_{n}$ is bounded by 0 and 1 uniformly on $[0, T]$, and so

$$
\begin{aligned}
\left|\bar{c}_{n+1}(t)\right|= & \mid G_{t}\left(\frac{\alpha_{t}}{\gamma_{t} \sigma_{t}^{2}}\left\{e^{-\int_{t}^{T}\left[r_{s}+\alpha_{s} c_{n}(s)+\sigma_{s}^{2} c_{n}^{2}(s)\right] d s}+\gamma_{t} e^{-\int_{t}^{T} \sigma_{s}^{2} c_{n}^{2}(s) d s}-\gamma_{t}\right\}\right) \\
& -G_{t}\left(\frac{\alpha_{t}}{\gamma_{t} \sigma_{t}^{2}}\left\{e^{-\int_{t}^{T}\left[r_{s}+\alpha_{s} c_{n-1}(s)+\sigma_{s}^{2} c_{n-1}^{2}(s)\right] d s}+\gamma_{t} e^{-\int_{t}^{T} \sigma_{s}^{2} c_{n-1}^{2}(s) d s}-\gamma_{t}\right\}\right) \mid \\
\leq & \left|\frac{\alpha_{t}}{\gamma_{t} \sigma_{t}^{2}}\right|\left(\left|e^{-\int_{t}^{T}\left[r_{s}+\alpha_{s} c_{n}(s)+\sigma_{s}^{2} c_{n}^{2}(s)\right] d s}-e^{-\int_{t}^{T}\left[r_{s}+\alpha_{s} c_{n-1}(s)+\sigma_{s}^{2} c_{n-1}^{2}(s)\right] d s}\right|\right. \\
& \left.+\gamma_{t}\left|e^{-\int_{t}^{T} \sigma_{s}^{2} c_{n}^{2}(s) d s}-e^{-\int_{t}^{T} \sigma_{s}^{2} c_{n-1}^{2}(s) d s}\right|\right) \\
\leq & K^{\prime}\left\{e^{(r+\alpha) T} \int_{t}^{T} \alpha\left|\bar{c}_{n}(s)\right|+\bar{\sigma}^{2}\left[c_{n}(s)+c_{n-1}(s)\right]\left|\bar{c}_{n}(s)\right| d s\right. \\
& \left.+\bar{\gamma} \int_{t}^{T} \bar{\sigma}^{2}\left[c_{n}(s)+c_{n-1}(s)\right]\left|\bar{c}_{n}(s)\right| d s\right\} \\
\leq & K^{\prime}\left(e^{(r+\alpha) T}\left(\alpha+2 \bar{\sigma}^{2}\right)+2 \bar{\gamma} \bar{\sigma}^{2}\right) \int_{t}^{T}\left|\bar{c}_{n}(s)\right| d s
\end{aligned}
$$

for all $t \in[0, T]$. Define $\phi_{n}(t):=\int_{t}^{T}\left|\bar{c}_{n}(s)\right| d s$. From (6.12), we have

$$
-\phi_{n}^{\prime}(t) \leq K \phi_{n-1}(t)
$$

which implies

$$
\phi_{n}(t)=-\int_{t}^{T} \phi_{n}^{\prime}(s) d s \leq K \int_{t}^{T} \phi_{n-1}(s) d s .
$$

By induction, we can deduce that

$$
\phi_{n+1}(t) \leq \frac{K^{n}}{n !}(T-t)^{n} \phi_{1}(0) .
$$

By (6.12) again, we have

$$
\left|\bar{c}_{n+1}(t)\right| \leq K \phi_{n}(t) \leq \frac{K^{n}}{(n-1) !}(T-t)^{n-1} \phi_{1}(0) .
$$

Then, given a large integer $n$, for any $m>n$, we have

$$
\left|c_{n}(t)-c_{m}(t)\right| \leq \sum_{k=n}^{\infty}\left|\bar{c}_{k+1}(t)\right| \leq \frac{(K T)^{n}}{(n-1) !} \phi_{1}(0)\left[1+\frac{K T}{n-K T}\right] .
$$

Copyright $\odot$ by SIAM. Unauthorized reproduction of this article is prohibited. 
Hence, we can conclude that $\left\{c_{n}(t)\right\}_{n \in \mathbb{N}}$ is a uniformly Cauchy sequence and $c$ is the uniform limit of $c_{n}$. Then taking the limit on both sides of (6.7), $c$ is the solution of (6.6). For the uniqueness, assume that $c_{1}$ and $c_{2}$ are the solution of (6.6). Again, $c_{1}$ and $c_{2}$ are bounded by 0 and 1 uniformly on $[0, T]$, and by using the same approach as in (6.12), we have

$$
\begin{aligned}
& \left|c_{1}(t)-c_{2}(t)\right| \\
= & \mid G_{t}\left(\frac{\alpha_{t}}{\gamma_{t} \sigma_{t}^{2}}\left\{e^{-\int_{t}^{T}\left[r_{s}+\alpha_{s} c_{1}(s)+\sigma_{s}^{2} c_{1}^{2}(s)\right] d s}+\gamma_{t} e^{-\int_{t}^{T} \sigma_{s}^{2} c_{1}^{2}(s) d s}-\gamma_{t}\right\}\right) \\
& -G_{t}\left(\frac{\alpha_{t}}{\gamma_{t} \sigma_{t}^{2}}\left\{e^{-\int_{t}^{T}\left[r_{s}+\alpha_{s} c_{2}(s)+\sigma_{s}^{2} c_{2}^{2}(s)\right] d s}+\gamma_{t} e^{-\int_{t}^{T} \sigma_{s}^{2} c_{2}^{2}(s) d s}-\gamma_{t}\right\}\right) \mid \\
\leq & K\left\{e^{(r+\alpha) T}\left|\int_{t}^{T} \alpha_{s}\left[c_{1}(s)-c_{2}(s)\right]+\sigma_{s}^{2}\left[c_{1}^{2}(s)-c_{2}^{2}(s)\right] d s\right|+\bar{\gamma}\left|\int_{t}^{T} \sigma_{s}^{2}\left[c_{1}^{2}(s)-c_{2}^{2}(s)\right] d s\right|\right\} \\
\leq & K \int_{t}^{T}\left|c_{1}(s)-c_{2}(s)\right| d s .
\end{aligned}
$$

Finally, Gronwall's inequality concludes that $c_{1}=c_{2}$ on $[0, T]$.

Next, we consider the convergence of the results from discrete time to that in the continuous time framework. The dynamic of wealth process in the discrete time model is

$$
X_{n+1}=\left(X_{n}-u_{n}\right) e^{\int_{t_{n}}^{t_{n}} r_{s} d s}+u_{n} e^{\int_{t_{n}}^{t_{n}}\left(\mu_{s}-\frac{1}{2} \sigma_{s}^{2}\right) d s+\int_{t_{n}}^{t_{n+1}} \sigma_{s} d W_{s}} .
$$

Here, the riskless return rate is $e^{\int_{t_{n}}^{t_{n+1}}} r_{s} d s$, and the risky return rate is $e^{\int_{t_{n}}^{t_{n+1}}\left(\mu_{s}-\frac{1}{2} \sigma_{s}^{2}\right) d s+\int_{t_{n}}^{t_{n+1}} \sigma_{s} d W_{s}}$. Take the coefficients $p_{n}, q_{n}, \gamma_{n}, r_{n}, \mu_{n}, \sigma_{n}$, and $\alpha_{n}$ as

$$
\begin{gathered}
p_{n}=p_{t_{n}}, \quad q_{n}=q_{t_{n}}, \quad \gamma_{n}=\gamma_{t_{n}}, \quad r_{n}=e^{\int_{t_{n}}^{t_{n}} r_{s} d s}, \quad \mu_{n}=e^{\int_{t_{n}}^{t_{n+1}} \mu_{s} d s}, \\
\sigma_{n}^{2}=\left(e^{\int_{t_{n}}^{t_{n}+1} \sigma_{s}^{2} d s}-1\right) e^{\int_{t_{n}}^{t_{n}} \mu_{s} d s}, \quad \alpha_{n}=e^{\int_{t_{n}}^{t_{n}} r_{s} d s}\left(e^{\int_{t_{n}}^{t_{n}+1} \alpha_{s} d s}-1\right) .
\end{gathered}
$$

Recall that the solution in the continuous time model is $\hat{u}(t, x)=c(t) x$, where $c(t)$ satisfies

$$
c(t)=G_{t}\left(\frac{\alpha_{t}}{\gamma_{t} \sigma_{t}^{2}}\left\{e^{-\int_{t}^{T}\left[r_{s}+\alpha_{s} c(s)+\sigma_{s}^{2} c^{2}(s)\right] d s}+\gamma_{t} e^{-\int_{t}^{T} \sigma_{s}^{2} c^{2}(s) d s}-\gamma_{t}\right\}\right) .
$$

Define $a(t)$ and $b(t)$ as follows:

$$
\begin{aligned}
a(t) & :=e^{\int_{t}^{T}\left[r_{s}+\alpha_{s} c(s)\right] d s} \\
b(t) & :=e^{2 \int_{t}^{T}\left[r_{s}+\alpha_{s} c(s)+\frac{1}{2} \sigma_{s}^{2} c^{2}(s)\right] d s} ;
\end{aligned}
$$

thus $a(t)$ and $b(t)$ are differentiable, and $a(t)$ and $b(t)$ are solutions to the ODE system

$$
\begin{aligned}
a^{\prime}(t)+\left(r_{t}+\alpha_{t} c(t)\right) a(t) & =0, \\
a(T) & =1 ; \\
b^{\prime}(t)+\left\{2\left(r_{t}+\alpha_{t} c(t)\right)+\sigma_{t}^{2} c(t)^{2}\right\} b(t) & =0, \\
b(T) & =1 .
\end{aligned}
$$

Copyright (C) by SIAM. Unauthorized reproduction of this article is prohibited. 
Since the coefficients of this ODE system are all bounded uniformly on $[0, T], a(t)$ and $b(t)$ are their unique solutions. Then $c(t)$ can be expressed in term of $a(t)$ and $b(t)$ :

$$
c(t)=G_{t}\left[\frac{\alpha_{t}}{\gamma_{t} \sigma_{t}^{2}} \frac{a(t)+\gamma_{t}\left[a(t)^{2}-b(t)\right]}{b(t)}\right] .
$$

We have the following convergent theorem, which states that $C_{n}$ as obtained in Theorem 4.1 converges to $c\left(t_{n}\right)$ as obtained in (6.6) as $\epsilon$ goes to 0 , where the coefficients in the discrete time model are given in (6.13). The above representation of $c(t)$ in terms of $a(t)$ and $b(t)$ can motivate the proof of this convergence result.

Theorem 6.4. For every fixed (small) $\epsilon>0$, if $c(t)$ is the solution of the integral equation (6.6) and $C_{n}$ are given as in Theorem 4.1 with the coefficients as defined in (6.13), then there exists $K>0$, independent of $n$, such that $\left|C_{n}-c\left(t_{n}\right)\right|<K \epsilon$.

The proof of Theorem 6.4 is given in Appendix B.

7. Numerical simulation. In this section, we compare the performance under different constraints on the investment to wealth ratio. In the first subsection, we investigate the effect of constraints, and we shall compare the equilibrium investment to wealth ratio and value function against $t$. In the second subsection, for different models of $\gamma(t)$, we shall compare the equilibrium investment to wealth ratio and value function at $t=0$ and $x=1$ against $T$. In the third subsection, we compare the mean-variance distribution representing the conditional expectation and conditional variance for varying risk-aversion coefficients. At the same time, we compare the performance of three investors: the first investor allows short selling (unconstrained investor), the second investor disallows short selling (constrained investor), and the third investor disallows short selling and invests at most half of his wealth into risky assets (conservative investor); the performance of an investor, who puts his wealth only in riskless assets, is augmented as a reference.

We set the risk-aversion coefficient $\gamma(t)$ to be time varying, as is usual for an investor being relatively less risk averse if the time to the expiry is still long, which we model $\gamma(t)$ by using the following logit function with known parameters $k_{1}$ and $k_{2}$ :

$$
\gamma(t ; T):=\frac{k_{1}}{1+e^{-k_{2}(t-T)}} .
$$

$\frac{k_{1}}{2}$ gives the risk-aversion coefficient when the time is at the expiry date and it is the maximum risk aversion. Larger $k_{2}$ will lead to more significant change in risk aversion as the time to the expiry is larger, so that the risk aversion of the long term investor will be smaller for larger $k_{2}$. Also, we fix $r=0.05, \mu=0.2$, and $\sigma=0.2$.

For the computation of investment to wealth ratio, the equilibrium value function, and the conditional expected value and conditional variance with equilibrium control for the unconstrained investor, refer to [4]. For the constrained investor, we first set $p_{t}=0$ and $q_{t}=1$, then the investment to wealth ratio can be found from $c(t)$ in (6.6), and the equilibrium value function is given in (6.3). The computation details for the conservative investor can similarly be found by setting $p_{t}=0$ and $q_{t}=0.5$.

Since obtaining the investment to wealth ratio $c(t)$ directly from (6.6) is not immediate,

Copyright $\odot$ by SIAM. Unauthorized reproduction of this article is prohibited. 
instead, the investment to wealth ratio, $c(t ; T)$, can be expressed as

$$
c(t ; T)=G\left[\frac{\alpha}{\gamma(t ; T) \sigma^{2}} \frac{a(t ; T)+\gamma(t ; T)\left[a(t ; T)^{2}-b(t ; T)\right]}{b(t ; T)}\right],
$$

where $a(t ; T)$ and $b(t ; T)$ are the solution for the following ODE system:

$$
\begin{aligned}
\frac{d a}{d t}(t ; T)+(r+\alpha c(t ; T)) a(t ; T) & =0, \\
a(T ; T) & =1 ; \\
\frac{d b}{d t}(t ; T)+\left\{2(r+\alpha c(t ; T))+\sigma^{2} c(t ; T)^{2}\right\} b(t ; T) & =0, \\
b(T ; T) & =1 .
\end{aligned}
$$

Thus $a(t ; T)$ and $b(t ; T)$ have the following expressions:

$$
\begin{aligned}
a(t ; T) & =e^{\int_{t}^{T}[r+\alpha c(s ; T)] d s}, \\
b(t ; T) & =e^{2 \int_{t}^{T}\left[r+\alpha c(s ; T)+\frac{1}{2} \sigma^{2} c^{2}(s ; T)\right] d s .} .
\end{aligned}
$$

At the same time, we can express the equilibrium value function and the conditional expected value and conditional variance with equilibrium control in terms of $a(t)$ and $b(t)$ :

$$
\begin{aligned}
V(t, x ; T) & =\left[a(t ; T)+\frac{\gamma(t ; T)}{2}\left(a(t ; T)^{2}-b(t ; T)\right)\right] x, \\
\mathbb{E}_{t, x}\left[X_{T}^{\hat{u}}\right] & =a(t ; T) x, \\
\operatorname{Var}_{t, x}\left[X_{T}^{\hat{u}}\right] & =\left(b(t ; T)-a(t ; T)^{2}\right) x^{2} .
\end{aligned}
$$

7.1. Effect of control constraints. In Figure $1, k_{1}=5, k_{2}=0.05, T=50$, all investors will invest more in risky assets when the time is closer to the expiry. Moreover, when the time is close to the expiry, the unconstrained investor will short sell riskless assets to invest more in risky assets to get the most satisfaction. At the same time, the constrained investor and the conservative investor are not allowed to invest too much in risky assets; their upper constraints are activated so that they can invest in risky assets only at their maximum levels. However, the highest risky investment for the unconstrained investor at the expiry makes his uncertainty at time 0 become the largest, so his value function at that time will be less than the constrained investor and the conservative investor. To hedge such a large uncertainty at the expiry, the unconstrained investor will short sell the risky asset when the time is far from the expiry, but the constrained investor and the conservative investor cannot short sell, so they can put all their wealth only on riskless assets.

7.2. Effect of different risk-aversion coefficient models. We first consider the case of large terminal risk aversion, so that all three risky investors will take the same strategy up to the expiry, and we fix $k_{1}=50$. In Figure 2, we further set $k_{2}=0.1$, large $k_{1}$ and small $k_{2}$ maintain large risk aversion, and all three risky investors maintain a steady investment. The unconstrained investor invests less than half of his wealth over the whole time horizon even though he is allowed to invest more in risky assets, so all three investors take the same

Copyright $\odot$ by SIAM. Unauthorized reproduction of this article is prohibited. 


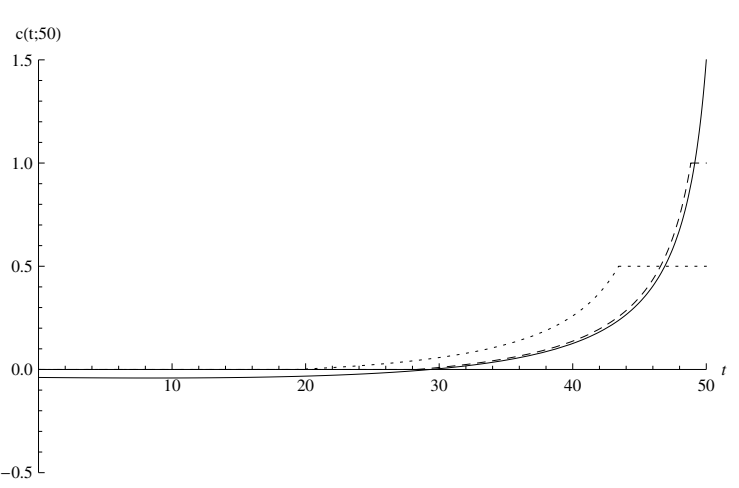

(a)

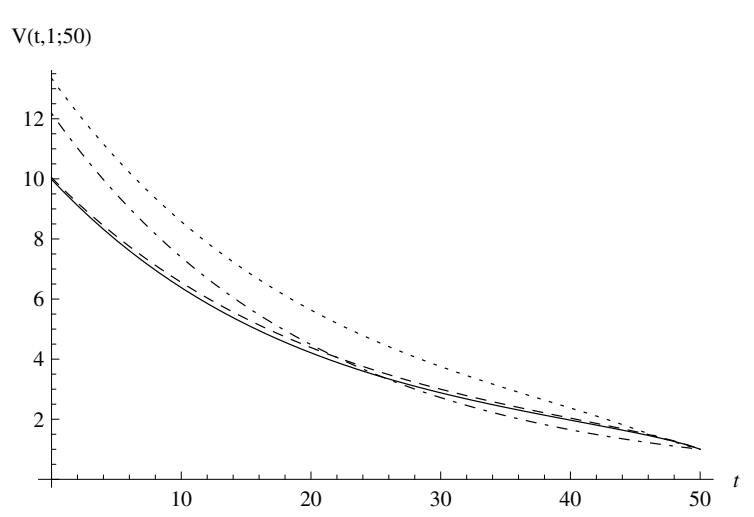

(b)

Figure 1. Fix $k_{1}=5, k_{2}=0.05, T=50, x=1$ : (a) The comparison between the investment to wealth ratio against $t$ for different investors. (b) The comparison between the equilibrium value functions against $t$ for different investors. Full line represents the unconstrained investor, dashed line represents the constrained investor, dotted line represents the conservative investor, and dotdashed line represents the riskless investor (available only in (b)).

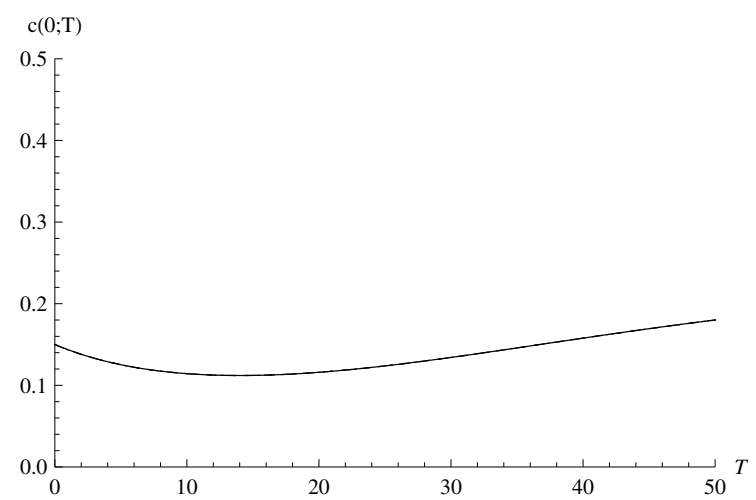

(a)

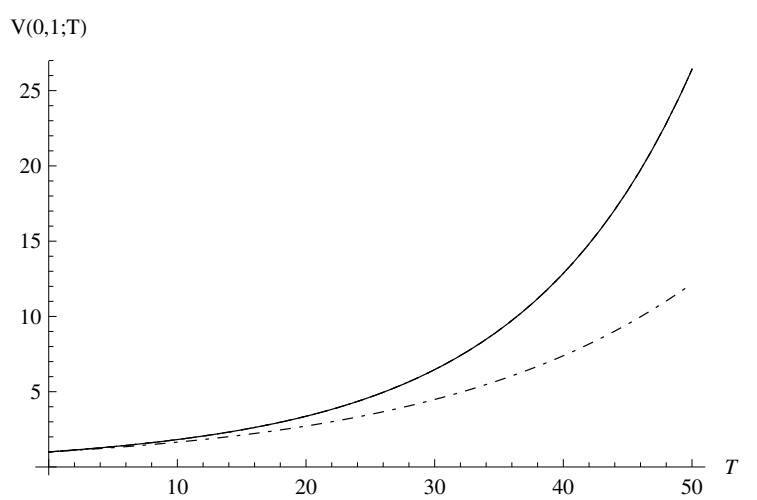

(b)

Figure 2. Fix $k_{1}=50, k_{2}=0.1, t=0, x=1$ : (a) The comparison between the investment to wealth ratio against $T$ for different investors. (b) The comparison between the equilibrium value functions against $T$ for different investors. Full line represents the unconstrained investor, dashed line represents the constrained investor, dotted line represents the conservative investor, and dotdashed line represents the riskless investor (available only in (b)).

strategy. Besides, they invest less risky in midterm investments, compared with those over the long and short terms, as they hedge out the risk over short term investments. For long term investments, the risk aversion is small, so they can invest more in risky assets.

In Figures 3 and 4, we increase $k_{2}$ to 0.2 and 0.3 , respectively; then the risk aversion for the long term investor will be smaller than that in Figure 2. Thus, the unconstrained investor will take more risky investments in the long term, and the constrained investor and the conservative investor will follow the unconstrained investor to carry out aggressive investments until the risky investments reach their maximum limit. Because of being forbidden to take too risky of 


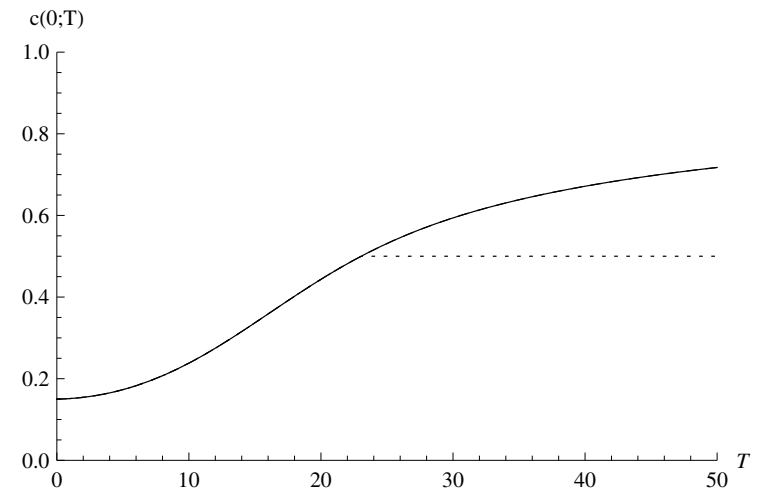

(a)

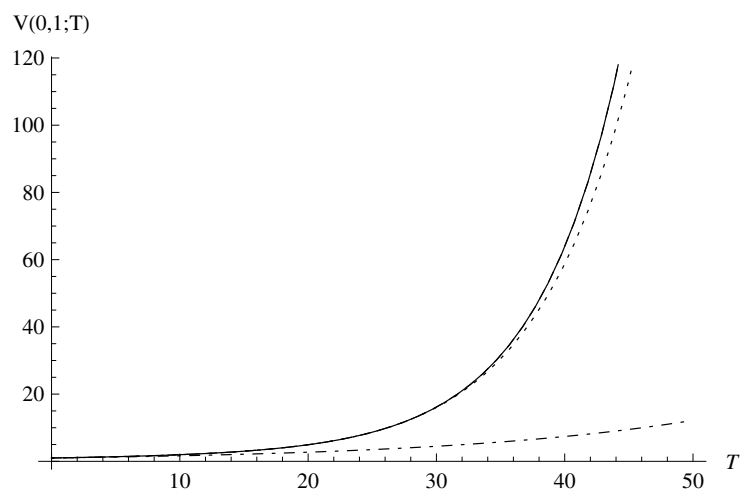

(b)

Figure 3. Fix $k_{1}=50, k_{2}=0.2, t=0, x=1$ : (a) The comparison between the investment to wealth ratio against $T$ for different investors. (b) The comparison between the equilibrium value functions against $T$ for different investors. Full line represents the unconstrained investor, dashed line represents the constrained investor, dotted line represents the conservative investor, and dotdashed line represents the riskless investor (available only in (b)).

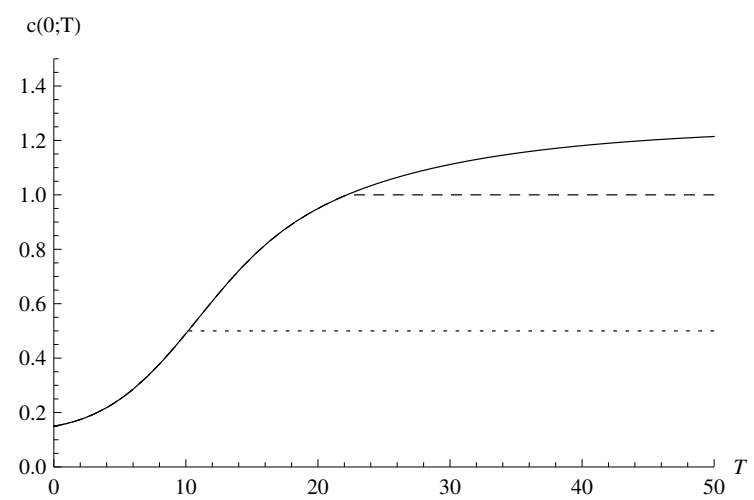

(a)

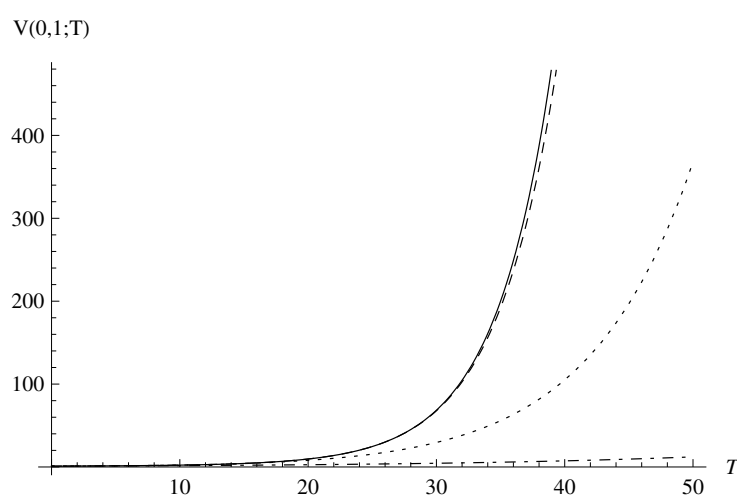

(b)

Figure 4. Fix $k_{1}=50, k_{2}=0.3, t=0, x=1$ : (a) The comparison between the investment to wealth ratio against $T$ for different investors. (b) The comparison between the equilibrium value functions against $T$ for different investors. Full line represents the unconstrained investor, dashed line represents the constrained investor, dotted line represents the conservative investor, and dotdashed line represents the riskless investor (available only in (b)).

investments, the performance of the constrained investor and the conservative investor will not be as good as that of the unconstrained investor since they cannot follow the unconstrained investor to take more risky investments.

Later, we set $k_{1}=1$ in Figures $5-7$, and thus the terminal risk aversion is small so that the unconstrained investor will short sell riskless assets at expiry. The long term investment strategy is similar to the case of $k_{1}=50$. The constrained investor and conservative investor cannot short sell, so they can just invest as many risky assets as they can. The unconstrained risky investments make the unconstrained investor able to perform better than the other two 


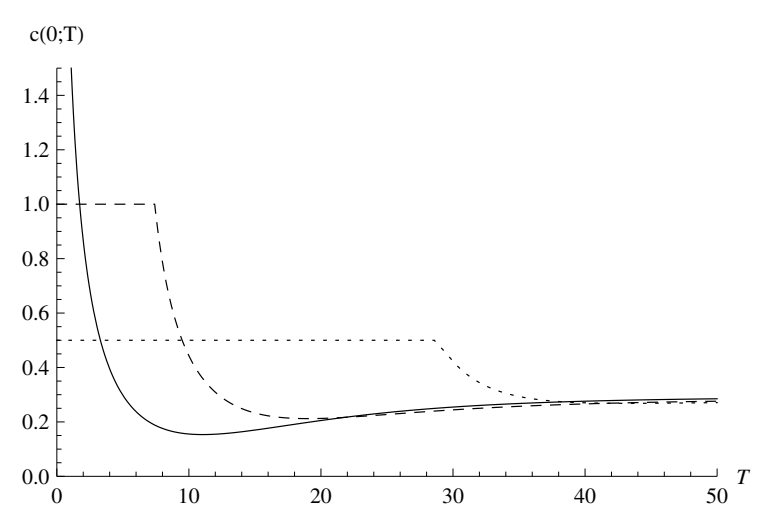

(a)

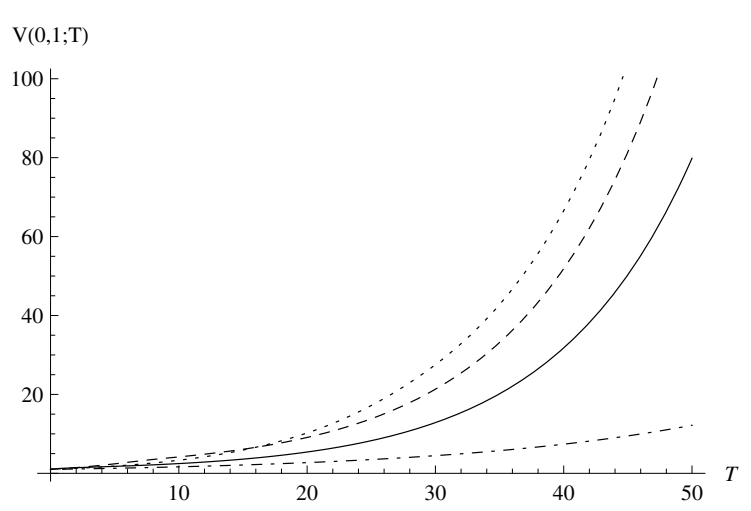

(b)

Figure 5. Fix $k_{1}=1, k_{2}=0.1, t=0, x=1$ : (a) The comparison between the investment to wealth ratio against $T$ for different investors. (b) The comparison between the equilibrium value functions against $T$ for different investors. Full line represents the unconstrained investor, dashed line represents the constrained investor, dotted line represents the conservative investor, and dotdashed line represents the riskless investor (available only in (b)).

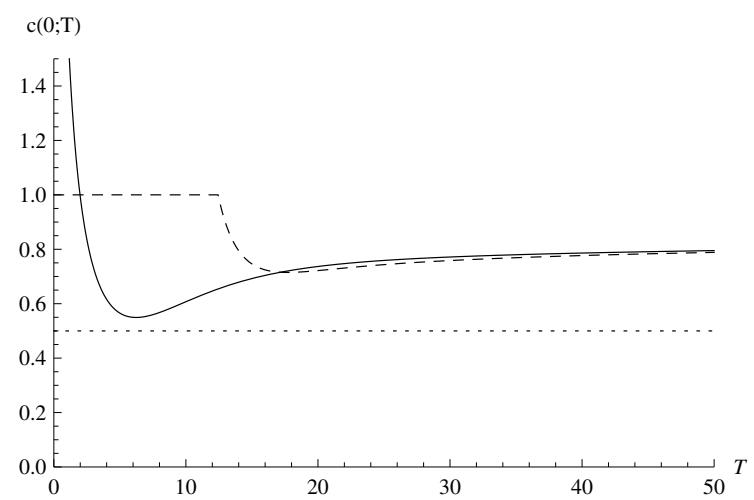

(a)

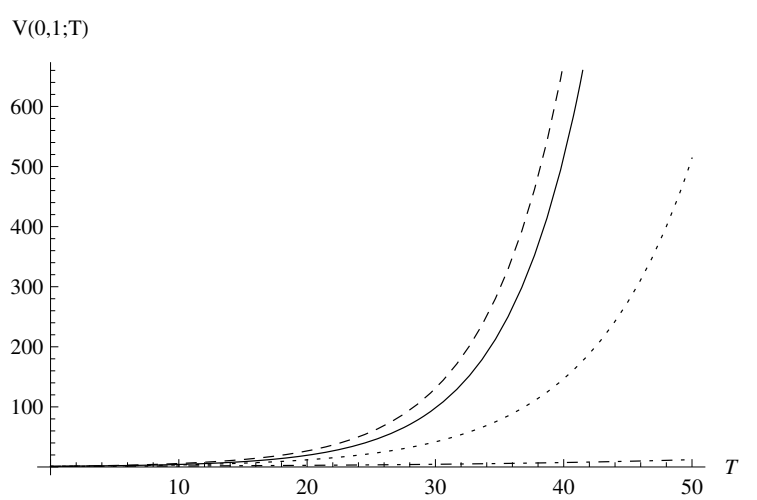

(b)

Figure 6. Fix $k_{1}=1, k_{2}=0.2, t=0, x=1$ : (a) The comparison between the investment to wealth ratio against $T$ for different investors. (b) The comparison between the equilibrium value functions against $T$ for different investors. Full line represents the unconstrained investor, dashed line represents the constrained investor, dotted line represents the conservative investor, and dotdashed line represents the riskless investor (available only in (b)).

risky investors in Tables 1 and 2. However, the huge risky investments in the short term result in a high volatility drawback in long term investments, and thus the conservative investors perform better than the other two risky investors in Figure 5.

In Figure 6, the constrained investor has the best long term performance among all three risky investors. It is because the excessive short term investments of the unconstrained investor cause large long term uncertainty, while the conservative investor cannot follow the other two risky investors to invest more in risky assets which causes his return to be less. In Figure 7, the allowance of long term unconstrained risky investments makes the unconstrained investor 


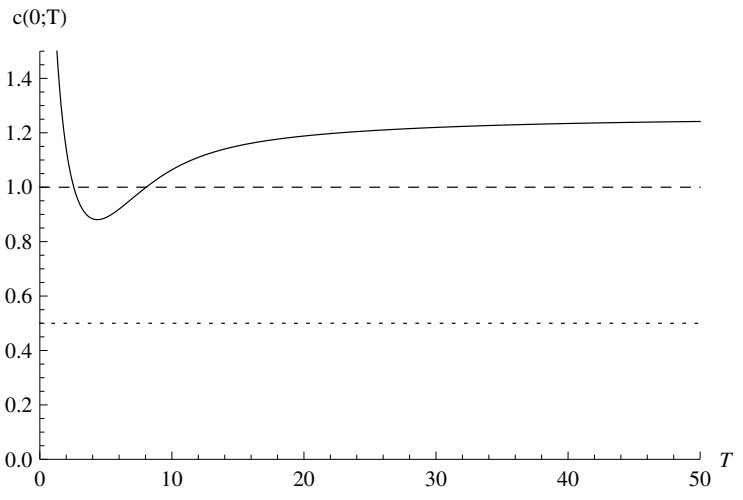

(a)

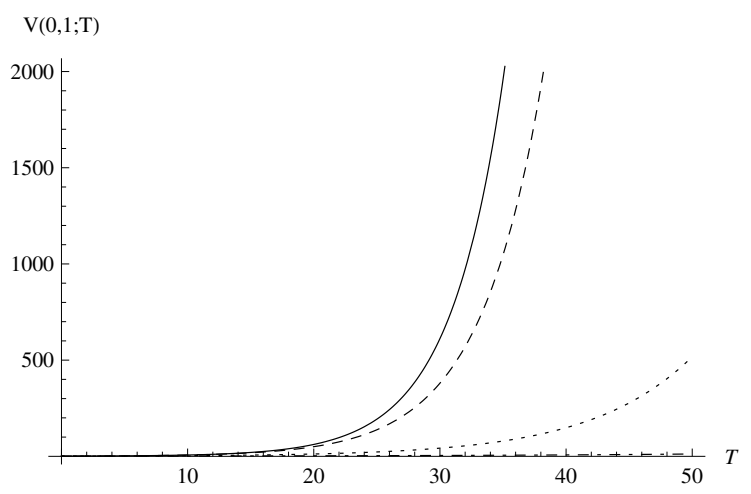

(b)

Figure 7. Fix $k_{1}=1, k_{2}=0.3, t=0, x=1$ : (a) The comparison between the investment to wealth ratio against $T$ for different investors. (b) The comparison between the equilibrium value functions against $T$ for different investors. Full line represents the unconstrained investor, dashed line represents the constrained investor, dotted line represents the conservative investor, and dotdashed line represents the riskless investor (available only in (b)).

Table 1

\begin{tabular}{|l|l|l|l|l|}
\hline \multicolumn{5}{|c|}{ Equilibrium utility value for different investors } \\
in different $T$ with $k_{1}=1, k_{2}=0.1$ when $t=0$ and $x=1$ \\
\hline Investor & Unconstrained & Constrained & Conservative & Riskless \\
\hline$T=1$ & $\mathbf{1 . 2 8 3 9}$ & 1.2069 & 1.1301 & 1.0513 \\
\hline$T=10$ & 2.4730 & $\mathbf{4 . 1 6 2 3}$ & 1.8345 & 1.6487 \\
\hline$T=20$ & 5.3994 & 9.0910 & $\mathbf{1 0 . 2 2 4 0}$ & 2.7183 \\
\hline$T=50$ & 78.8514 & 128.2294 & $\mathbf{1 6 2 . 7 8 6 0}$ & 12.1825 \\
\hline
\end{tabular}

Table 2

\begin{tabular}{|l|l|l|l|l|}
\hline \multicolumn{5}{|c|}{ Equilibrium utility value for different investors } \\
in different $T$ with $k_{1}=1, k_{2}=0.2$ when $t=0$ and $x=1$ \\
\hline Investor & Unconstrained & Constrained & Conservative & Riskless \\
\hline$T=1$ & $\mathbf{1 . 3 0 0 4}$ & 1.2077 & 1.1302 & 1.0513 \\
\hline$T=10$ & 4.1916 & $\mathbf{5 . 7 8 8 6}$ & 3.4140 & 1.6487 \\
\hline$T=20$ & 19.3953 & $\mathbf{2 6 . 6 3 5 1}$ & 11.8870 & 2.7183 \\
\hline$T=50$ & 2754.3159 & $\mathbf{3 5 4 5 . 4 2 4 3}$ & 514.0615 & 12.1825 \\
\hline
\end{tabular}

outperform the other two risky investors.

In conclusion, the unconstrained investor, constrained investor, and conservative investor can perform better than the other two in a certain value of $k_{1}, k_{2}$, and $T$. We observe that the long term investments of three risky investors are steady; larger $k_{2}$ will make the unconstrained investor have more risky long term investments because of smaller long term risk aversion. However, if any investor cannot follow the long term investments of the unconstrained investor because of the constraint, then his investment performance will be affected. Smaller $k_{1}$ encourages more risky investments in the short term, but it gives a drawback over long term investment performance because of the volatility, and thus the unconstrained investor will be 
significantly affected by this excessive risky investment in the short term. For further analysis about the nontypical observation that the constrained investor outperforms the unconstrained investor, Remark 5.4.1 in [29] provides a deep discussion in both mathematical and economical aspects.

7.3. Mean-variance distribution between different control constraints. In Figures $8-10$, we compare the mean-variance distribution for different investors setting $T=5,50$. We model the risk-aversion coefficient by the logit function with free varying $k_{1}, k_{2}$, and thus we can see a two-dimensional area of mean-variance distribution. We can see that the distribution area of the conservative investor is inside the distribution area of the constrained investor, but they occupy the most efficient part of the distribution area of the constrained investor. A similar relation appears between the constrained investor and unconstrained investor. Three investors share the same mean-variance distribution for small variance because they adopt the same investment strategy if the risk-aversion coefficient is large.

In Figures 8(a)-9, we see that the constrained investor and conservative investor are restricted by the upper bound in expected return because of constrained investment in risky assets, which explains the more conservative investor not performing well for small $T$.

It is also a surprise that the lower end of the mean-variance distribution of the unconstrained investor will have a shape of " 2 " for $T=50$.

Note that [11] found the same observation that the constrained investor adopts a more efficient strategy than that of the unconstrained investor.

8. Conclusion. In this paper, we studied mean-variance portfolio selection with shortselling prohibition via the time-consistent approach. For the present case that the risk aversion is inversely proportional to the current wealth, positive wealth assumption is required for the investor to remain risk averse, and so the short selling has to be prohibited (Remark 2.6). By using backward induction, the equilibrium control in the discrete time setting is shown to be linear in wealth where its coefficients can be obtained by backward recursion (Theorem 4.1). By using the extended HJB equation (Theorem 5.1), we showed that the equilibrium control in the continuous time setting is also linear in wealth with coefficients satisfying the integral equation in (Theorem 6.1); this integral equation has been shown to admit a unique solution (Theorem 6.2). We also show that the equilibrium control in the discrete time setting converges to that in the continuous time model (Theorem 6.4). Finally, some numerical comparisons of the performance for different control constraint were made.

Further extensions of our present problem could be investigated. First, one can consider the same problem with multiple risky assets; the corresponding feasible set of admissible controls is then confined to a multidimensional cone in light of the requirement of shortselling prohibition. Second, it is interesting to also consider the same problem with random parameters, but the model may not be Markovian anymore, and thus the approach in [3] cannot be directly applied. Instead, one can consider the work of [14], in which all adapted controls are regarded as admissible controls, and hence the corresponding problem could probably be resolved; finally, it may also be crucial to compare the performances of the respective equilibrium controls among different admissible sets, [3] and [14], as we can perceive that the equilibrium control over the smaller admissible set can outperform the corresponding

Copyright (c) by SIAM. Unauthorized reproduction of this article is prohibited. 


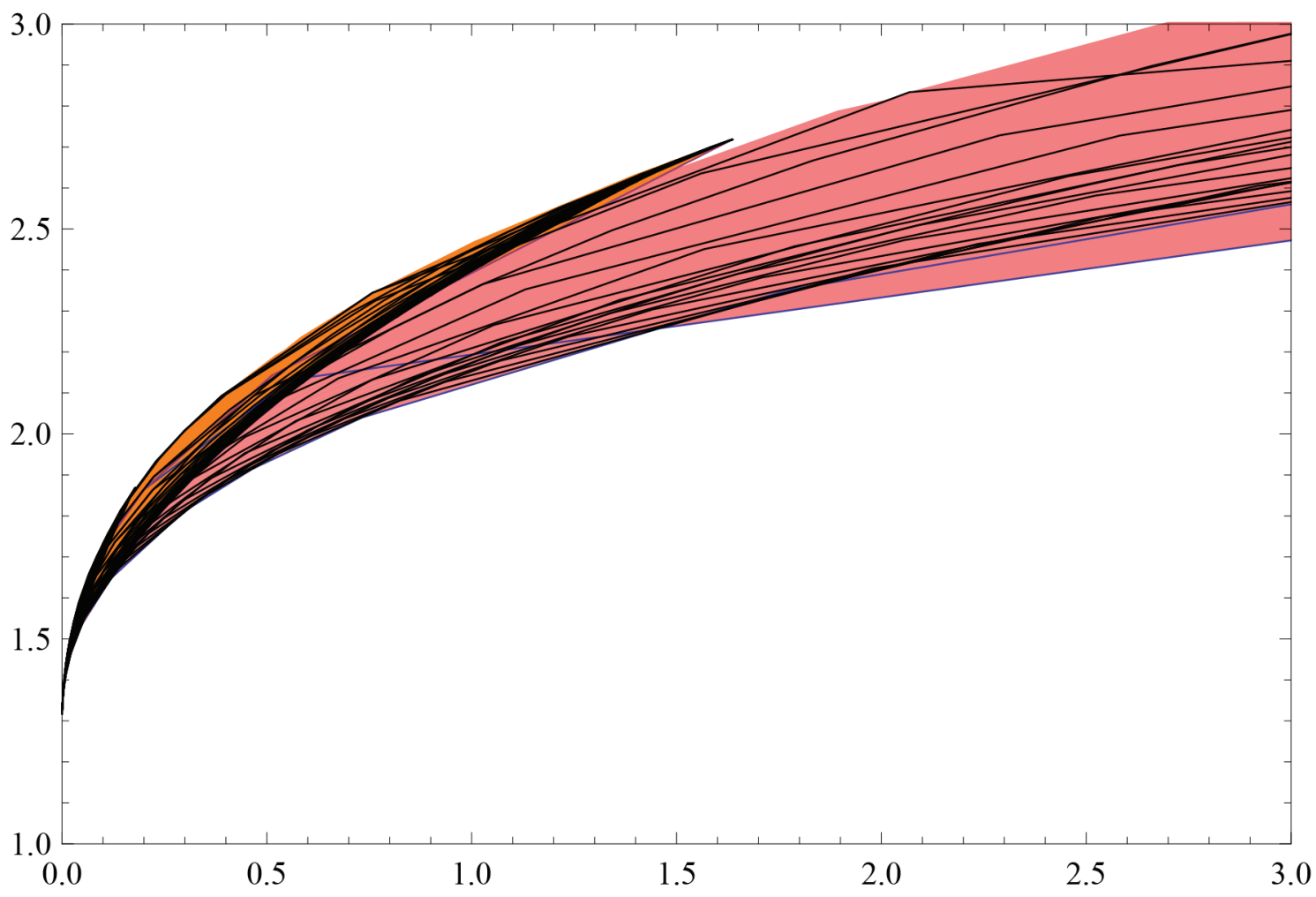

(a)

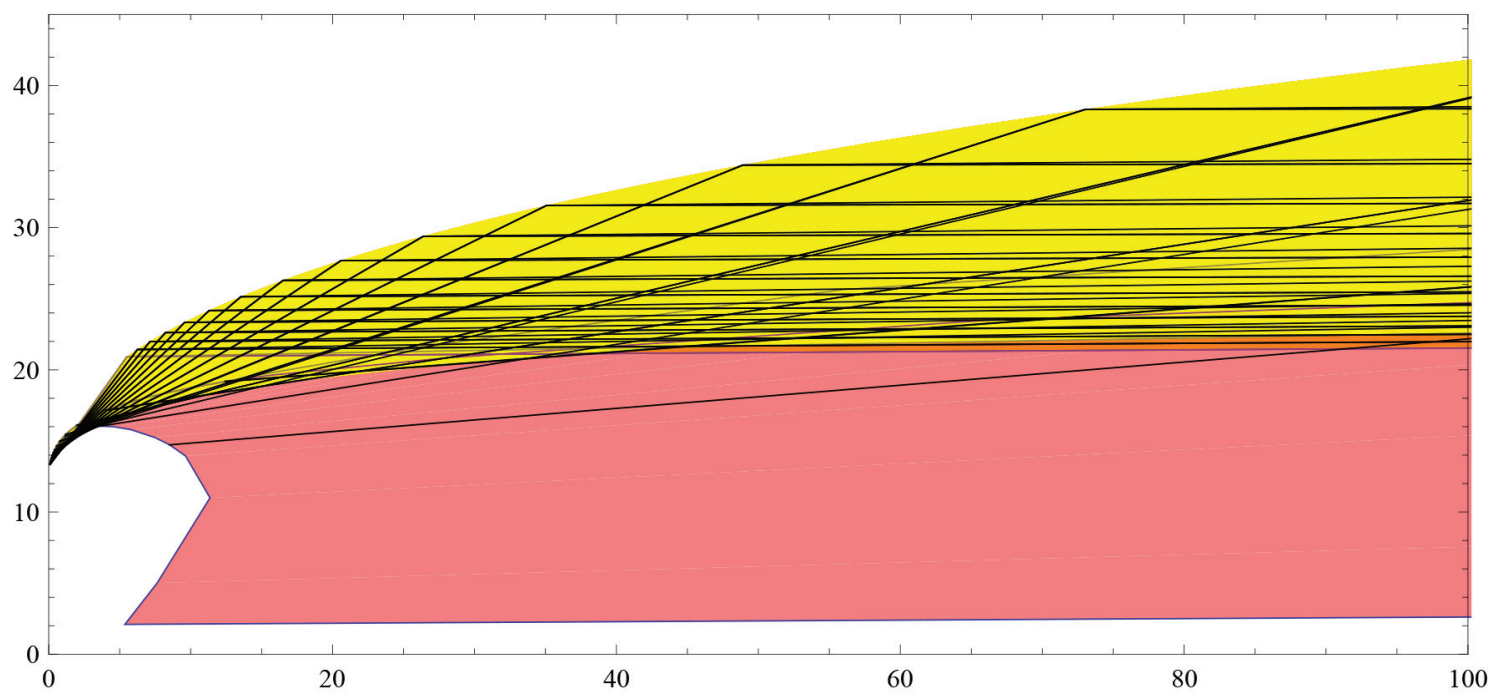

(b)

Figure 8. The comparison between the mean-variance distribution for different constraints with different expiry times $T$ : (a) $T=5$, (b) $T=50$, given the current time $t=0$ and current wealth $x=1$. The $y$-axis represents the equilibrium conditional expected return, and the $x$-axis represents the equilibrium conditional variance. Red area represents the unconstrained investor, orange area represents the constrained investor, and yellow area represents the conservative investor.

Copyright (C) by SIAM. Unauthorized reproduction of this article is prohibited. 


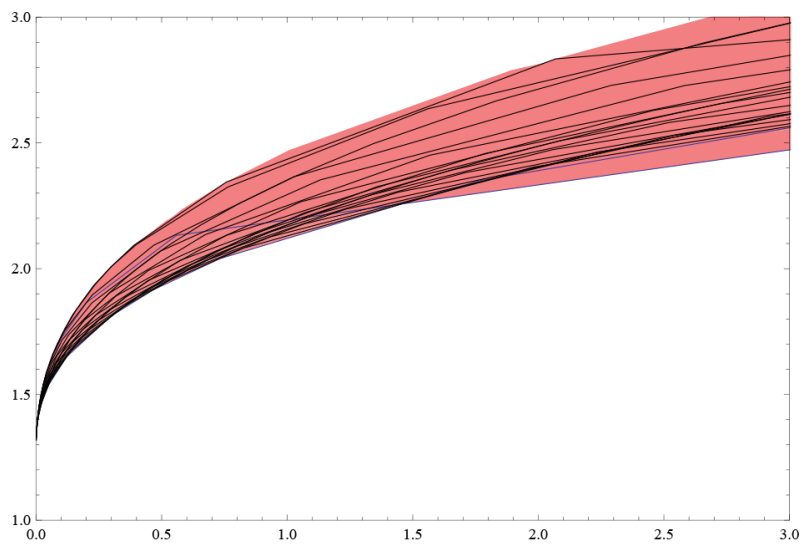

(a)

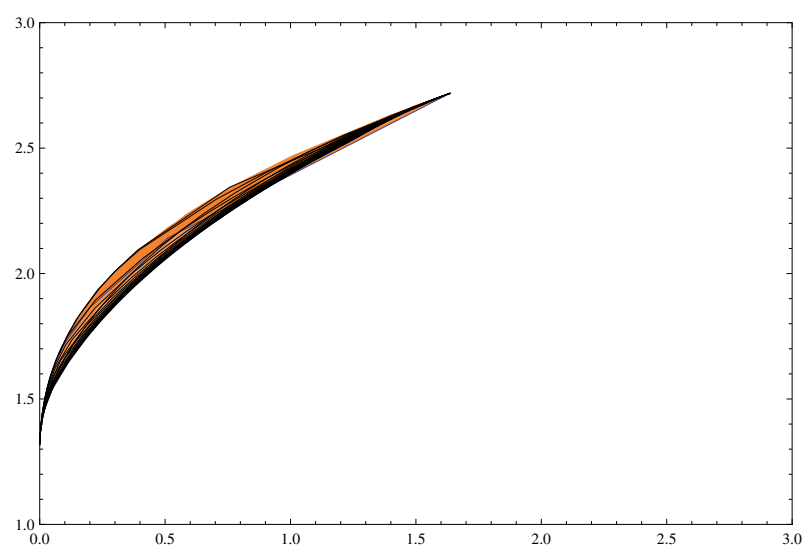

(b)

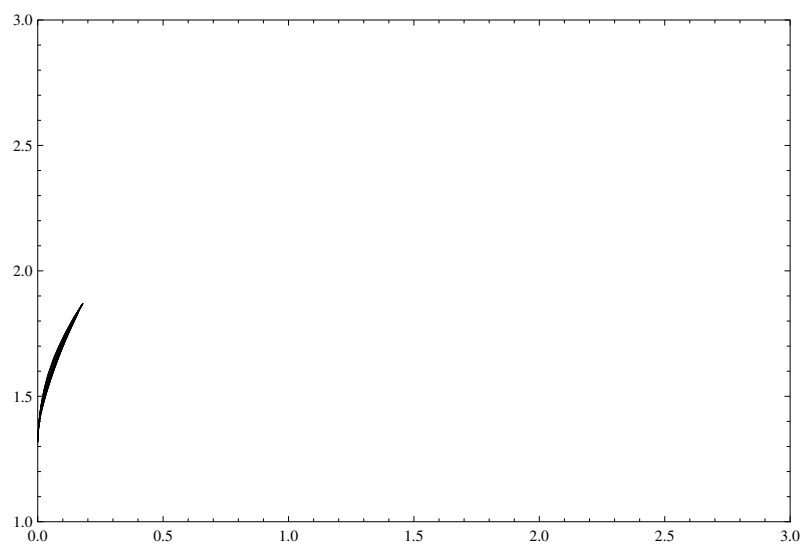

(c)

Figure 9. The mean-variance distribution for different constraints: (a) unconstrained investor, (b) constrained investor, (c) conservative investor, given the expiry $T=5$, the current time $t=0$, and current wealth $x=1$. The $y$-axis represents the equilibrium conditional expected return, and the $x$-axis represents the equilibrium conditional variance.

Copyright (C) by SIAM. Unauthorized reproduction of this article is prohibited. 


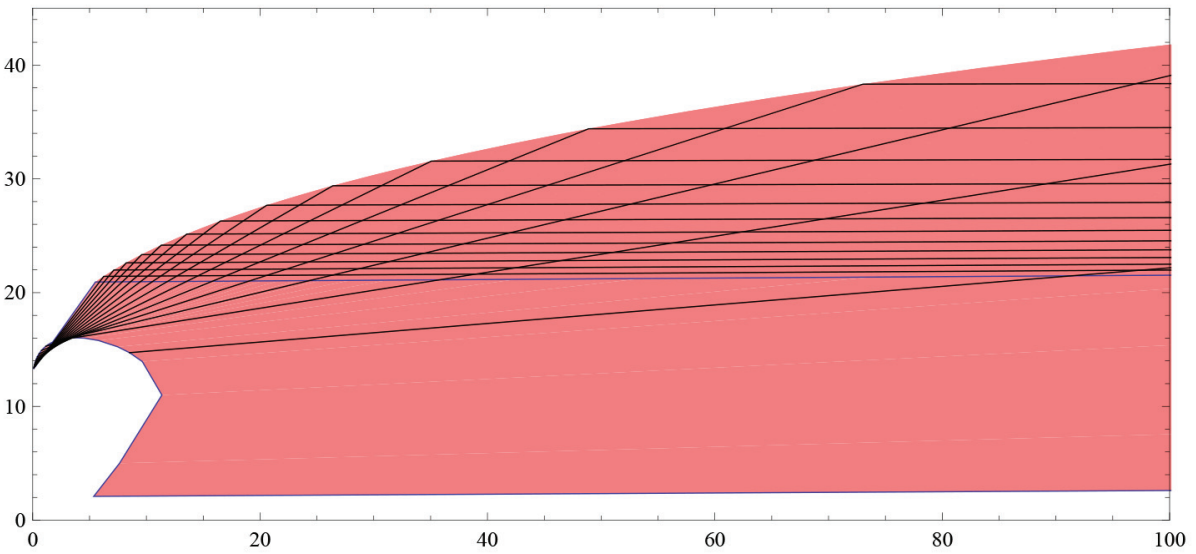

(a)

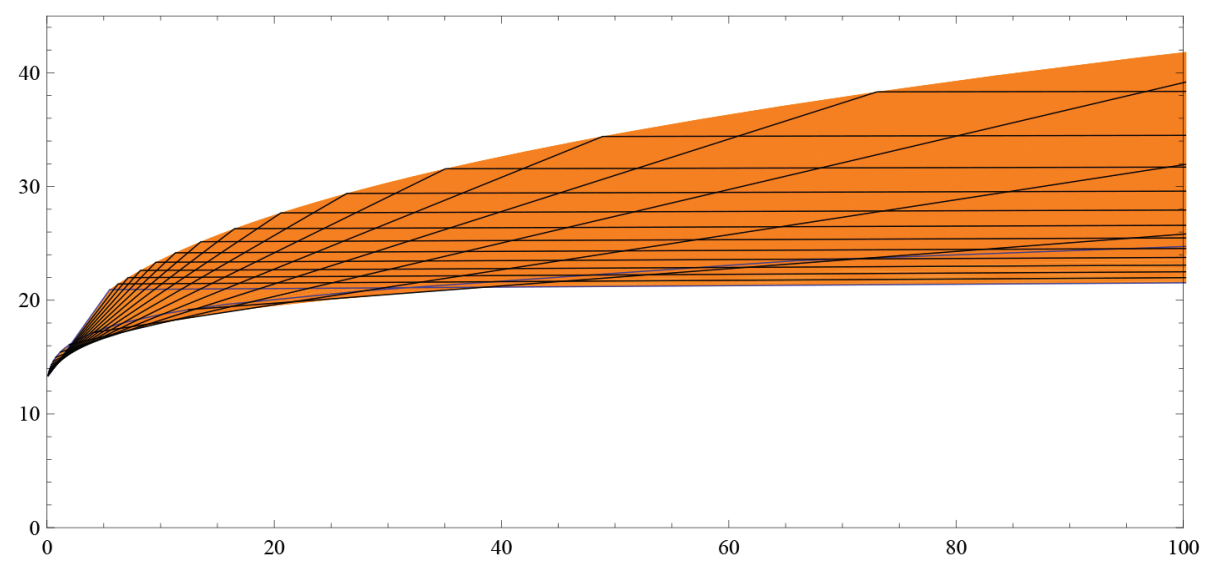

(b)

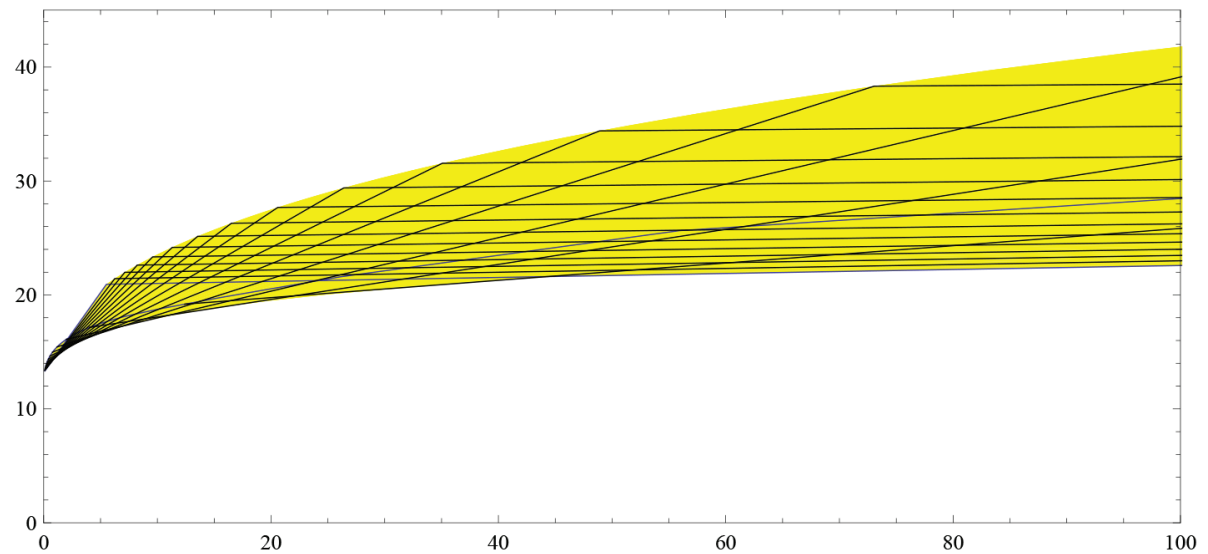

(c)

Figure 10. The mean-variance distribution for different constraints: (a) unconstrained investor, (b) constrained investor, (c) conservative investor, given the expiry $T=50$, the current time $t=0$, and current wealth $x=1$. The $y$-axis represents the equilibrium conditional expected return, and the $x$-axis represents the equilibrium conditional variance.

Copyright (C) by SIAM. Unauthorized reproduction of this article is prohibited. 
one over the larger one.

Appendix A. The proof of Theorem 5.1 and the relation between Theorem 5.1 and equilibrium control. In this section, we prove Theorem 5.1 by using the same approach as in Appendix A in [4], with the admissible set of $u$ taking values being confined in $\left[p_{t} x, q_{t} x\right]$ $($ rather than in $\mathbb{R})$ in the present short-selling prohibition model.

Proof of Theorem 5.1. Define

$$
G(t, x, y):=\frac{\gamma_{t}}{2 x} y^{2}
$$

According to the extended HJB system and the corresponding verification theorem given by [3], if we could find $V, g, \hat{u}:[0, T] \times \mathbb{R}^{+} \rightarrow \mathbb{R}, f:[0, T] \times \mathbb{R}^{+} \times[0, T] \times \mathbb{R}^{+} \rightarrow \mathbb{R}$ such that they satisfy the following extended HJB system, then we can get the conclusion of Theorem 5.1:

$$
\begin{aligned}
\sup _{u \in\left[p_{t} x, q_{t} x\right]}\left\{\left(\mathbf{A}^{u} V\right)(t, x)-\left(\mathbf{A}^{u} f\right)(t, x, t, x)+\left(\mathbf{A}^{u} f^{t, x}\right)(t, x)\right. & \\
\left.-\mathbf{A}^{u}(G \diamond g)(t, x)+\left(\mathbf{H}^{u} g\right)(t, x)\right\} & =0, \\
\left(\mathbf{A}^{\hat{u}} f^{s, y}\right)(t, x) & =0, \\
\left(\mathbf{A}^{\hat{u}} g\right)(t, x) & =0, \\
V(T, x) & =x, \\
f(T, x, s, y) & =x-\frac{\gamma_{s}}{2 y} x^{2}, \\
g(T, x) & =x,
\end{aligned}
$$

where the supremum in the first equation is attained at $\hat{u}(t, x)$ for all $x \in \mathbb{R}^{+}, \mathbf{A}^{\mathbf{u}}$ denotes the controlled infinitesimal generator, and $f^{s, y}, G \diamond g$, and $\mathbf{H}^{u} g$ are defined as

$$
\begin{aligned}
f^{s, y}(t, x) & =f(t, x, s, y), \\
(G \diamond g)(t, x) & =G(t, x, g(t, x)), \\
\mathbf{H}^{u} g(t, x) & =\left.\frac{\partial G}{\partial y}(t, x, y)\right|_{y=g(t, x)} \cdot \mathbf{A}^{u} g(t, x) .
\end{aligned}
$$

In reference to the dynamics of the controlled wealth process, we have the following expression for the infinitesimal operator:

$$
\mathbf{A}^{u}=\frac{\partial}{\partial t}+\left(r_{t} x+\alpha_{t} u\right) \frac{\partial}{\partial x}+\frac{1}{2} \sigma_{t}^{2} u^{2} \frac{\partial^{2}}{\partial x^{2}} .
$$

Thus, we have (5.5) to (5.9). Next, we make further computation on the first equation of the extended HJB system term by term:

$$
\begin{aligned}
\mathbf{A}^{u} V(t, x) & =\frac{\partial V}{\partial t}(t, x)+\left(r_{t} x+\alpha_{t} u\right) \frac{\partial V}{\partial x}(t, x)+\frac{1}{2} \sigma_{t}^{2} u^{2} \frac{\partial^{2} V}{\partial x^{2}}(t, x), \\
\mathbf{A}^{u} f^{t, x}(t, x) & =\frac{\partial f}{\partial t}(t, x, t, x)+\left(r_{t} x+\alpha_{t} u\right) \frac{\partial f}{\partial x}(t, x, t, x)+\frac{1}{2} \sigma_{t}^{2} u^{2} \frac{\partial^{2} f}{\partial x^{2}}(t, x, t, x), \\
\mathbf{A}^{u} f(t, x, t, x) & =\frac{\partial f}{\partial t}(t, x, t, x)+\frac{\partial f}{\partial s}(t, x, t, x)+\left(r_{t} x+\alpha_{t} u\right)\left(\frac{\partial f}{\partial x}(t, x, t, x)+\frac{\partial f}{\partial y}(t, x, t, x)\right)
\end{aligned}
$$

Copyright (C by SIAM. Unauthorized reproduction of this article is prohibited. 


$$
\begin{aligned}
& +\frac{1}{2} \sigma_{t}^{2} u^{2}\left(\frac{\partial^{2} f}{\partial x^{2}}(t, x, t, x)+\frac{\partial^{2} f}{\partial y^{2}}(t, x, t, x)+2 \frac{\partial^{2} f}{\partial x \partial y}(t, x, t, x)\right), \\
\mathbf{A}^{u}(G \diamond g)(t, x)= & \frac{\gamma_{t}^{\prime}}{2 x} g^{2}(t, x)+\frac{\gamma_{t}}{x} g(t, x) \frac{\partial g}{\partial t}(t, x) \\
& +\left(r_{t} x+\alpha_{t} u\right)\left(-\frac{\gamma_{t}}{2 x^{2}} g^{2}(t, x)+\frac{\gamma_{t}}{x} g(t, x) \frac{\partial g}{\partial x}(t, x)\right) \\
& +\frac{1}{2} \sigma_{t}^{2} u^{2}\left\{\frac{\gamma_{t}}{x^{3}} g^{2}(t, x)-2 \frac{\gamma_{t}}{x^{2}} g(t, x) \frac{\partial g}{\partial x}(t, x)+\frac{\gamma_{t}}{x}\left(\frac{\partial g}{\partial x}(t, x)\right)^{2}\right\} \\
& +\frac{1}{2} \sigma_{t}^{2} u^{2} \frac{\gamma_{t}}{x} g(t, x) \frac{\partial^{2} g}{\partial x^{2}}(t, x), \\
\mathbf{H}^{u} g(t, x)= & \frac{\gamma_{t}}{x} g(t, x)\left(\frac{\partial g}{\partial t}(t, x)+\left(r_{t} x+\alpha_{t} u\right) \frac{\partial g}{\partial x}(t, x)+\frac{1}{2} \sigma_{t}^{2} u^{2} \frac{\partial^{2} g}{\partial x^{2}}(t, x)\right) .
\end{aligned}
$$

Based on these expressions, we deduce (5.4).

Appendix B. Convergence of equilibrium controls in discrete time to those in continuous time. We now aim to establish Theorem 6.4. First, we prove the following Theorem B.5 that $A_{n}$ and $B_{n}$ in Theorem 4.1 with the coefficients as specified in (6.13) converge, respectively, to $a(t)$ and $b(t)$ as defined in (6.14). The present proof of the convergence is inspired by $[9]$.

Lemma B.1.

(a) Let $c(t)$ be the solution of the integral equation (6.6). If $a(t)$ and $b(t)$ are defined in (6.14), then there exist $k_{1}>0$ and $K_{1}<\infty$, which are independent of $\epsilon$, such that both $a(t)$ and $b(t)$ are bounded above by $K_{1}$ and bounded below by $k_{1}$ uniformly on $[0, T]$.

(b) Let $A_{n}$ and $B_{n}$ be given in Theorem 4.1 with coefficients as specified in (6.13); there exist $k_{2}>0$ and $K_{2}<\infty$ such that $B_{n}$ is bounded above by $K_{2}$ and bounded below by $k_{2}$ uniformly for all $n$ and $A_{n}$ is bounded above by $K_{2}$ uniformly for all $n$.

Proof. (a) Since $G_{t}$ is bounded by 0 and $1, c(t)$ is bounded by 0 and 1 uniformly on $[0, T]$. Since $r_{t}, \alpha_{t}$, and $\sigma_{t}$ are Lipschitz continuous, $r_{t}, \alpha_{t}$, and $\sigma_{t}$ are bounded uniformly on $[0, T]$ by $r, \alpha$, and $\bar{\sigma}$ as in (6.8). We have

$$
\begin{aligned}
0<e^{-(r+\alpha) T} & \leq a(t) \leq e^{(r+\alpha) T} \\
0<e^{-2(r+\alpha) T} & \leq b(t) \leq e^{\left(2 r+2 \alpha+\bar{\sigma}^{2}\right) T} .
\end{aligned}
$$

(b) By (4.4)-(4.7) in Theorem 4.1, we have $A_{n}=\prod_{k=n}^{N-1}\left(r_{k}+\alpha_{k} C_{k}\right)$ and $B_{n}=\prod_{k=n}^{N-1}\left[\left(r_{k}+\right.\right.$ $\left.\left.\alpha_{k} C_{k}\right)^{2}+\sigma_{k}^{2} C_{k}^{2}\right]$. Note that $C_{k}$ is bounded by 0 and 1 , so we have

$$
r_{k}+\alpha_{k} C_{k}=e^{\int_{t_{k}}^{t_{k+1}} r_{s} d s}\left[1+\left(e^{\int_{t_{k}}^{t_{k+1}} \alpha_{s} d s}-1\right) C_{k}\right] \leq e^{(r+\alpha) \epsilon}
$$

Thus, $A_{n}$ is bounded above uniformly:

$$
A_{n} \leq \prod_{k=n}^{N-1} e^{(r+\alpha) \epsilon}=e^{(r+\alpha) \sum_{k=n}^{N-1} \epsilon} \leq e^{(r+\alpha) T} .
$$

Copyright (C) by SIAM. Unauthorized reproduction of this article is prohibited. 
Similarly, note that $r_{k}+\alpha_{k} C_{k} \geq 0$, and so $\left(r_{k}+\alpha_{k} C_{k}\right)^{2} \leq e^{2(r+\alpha) \epsilon}$,

$$
\left(r_{k}+\alpha_{k} C_{k}\right)^{2}+\sigma_{k}^{2} C_{k}^{2} \leq e^{2(r+\alpha) \epsilon}+\left(e^{\bar{\sigma}^{2} \epsilon}-1\right) e^{2(r+\alpha) \epsilon}=e^{\left[2(r+\alpha)+\bar{\sigma}^{2}\right] \epsilon} .
$$

On the other hand, by completing the square in $C_{k}$,

$$
\left(r_{k}+\alpha_{k} C_{k}\right)^{2}+\sigma_{k}^{2} C_{k}^{2}=\left(\alpha_{k}^{2}+\sigma_{k}^{2}\right) C_{k}^{2}+2 r_{k} \alpha_{k} C_{k}+r_{k}^{2} \geq \frac{r_{k}^{2}}{1+\frac{\alpha_{k}^{2}}{\sigma_{k}^{2}}}
$$

We claim that $\frac{\alpha_{k}^{2}}{\sigma_{k}^{2}}$ is bounded above by $K \epsilon$ with a constant $K>0$ which is independent of $\epsilon$ :

$\frac{\alpha_{k}^{2}}{\sigma_{k}^{2}}=\frac{\left(e^{\int_{t_{n}}^{t_{n+1}} \alpha_{s} d s}-1\right)^{2}}{\left(e^{\int_{t_{n}}^{t_{n+1}} \sigma_{s}^{2} d s}-1\right)} e^{-2 \int_{t_{n}}^{t_{n}+1} \alpha_{s} d s} \leq \frac{\left(e^{\alpha \epsilon}-1\right)^{2}}{e^{\zeta} \cdot \int_{t_{n}}^{t_{n+1}} \sigma_{s}^{2} d s} e^{-2 \int_{t_{n}}^{t_{n}+1} \alpha_{s} d s} \leq \frac{\left(\alpha \epsilon e^{\alpha \eta}\right)^{2}}{\epsilon \underline{\sigma}^{2}} e^{2 \alpha \epsilon} \leq \epsilon \frac{\alpha^{2}}{\underline{\sigma}^{2}} e^{4 \alpha T}$

for some $\zeta \in\left[0, \int_{t_{n}}^{t_{n+1}} \sigma_{s}^{2} d s\right]$ and $\eta \in[0, \epsilon]$ in light of the mean value theorem. Therefore,

$$
\begin{aligned}
B_{n} & \leq \prod_{k=n}^{N-1} e^{\left[2(r+\alpha)+\bar{\sigma}^{2}\right] \epsilon} \leq e^{\left[2(r+\alpha)+\bar{\sigma}^{2}\right] T}, \\
B_{n} & \geq \prod_{k=n}^{N-1} e^{-2 r \epsilon} \frac{1}{1+\epsilon \frac{\alpha^{2}}{\underline{\sigma}^{2}} e^{4 \alpha T}}=e^{-2 r T} \frac{1}{\left[1+\frac{T}{N} \frac{\alpha^{2}}{\sigma^{2}} e^{4 \alpha T}\right]^{N-n}} \\
& \geq e^{-2 r T} \frac{1}{\lim _{N \rightarrow \infty}\left[1+\frac{T}{N} \frac{\alpha^{2}}{\underline{\sigma}^{2}} e^{4 \alpha T}\right]^{N}}=e^{-2 r T} e^{-\frac{\alpha^{2}}{\sigma^{2}} e^{4 \alpha T} T}>0 .
\end{aligned}
$$

Lemma B.2. If $c(t)$ is the solution of the integral equation (6.6), $c(t)$ is Lipschitz continuous.

Proof. Define $d(t):=e^{-\int_{t}^{T}\left[r_{s}+\alpha_{s} c(s)+\sigma_{s}^{2} c^{2}(s)\right] d s}+\gamma_{t} e^{-\int_{t}^{T} \sigma_{s}^{2} c^{2}(s) d s}-\gamma_{t}$. For any $s, t \in[0, T]$,

$|c(t)-c(s)|=\left|G_{t}\left(\frac{\alpha_{t}}{\gamma_{t} \sigma_{t}^{2}} d(t)\right)-G_{s}\left(\frac{\alpha_{s}}{\gamma_{s} \sigma_{s}^{2}} d(s)\right)\right| \leq K|d(t)||t-s|+K|d(t)-d(s)|+K|t-s|$.

Lipschitz continuity of $c$ follows if $d(t)$ can be shown to be uniformly bounded and Lipschitz continuous. With the same approaches as for (6.10) and (6.11), we can prove that $d(t)$ is uniformly bounded: $-\bar{\gamma} \leq d(t) \leq e^{(r+\alpha) T}+\bar{\gamma}$, and is also Lipschitz continuous.

Lemma B.3. Fix an $\epsilon>0$. $a(t)$ and $b(t)$ are defined as in (6.14), $c(t)$ satisfies (6.6), and $A_{n}, B_{n}$, and $C_{n}$ are given in Theorem 4.1 with coefficients as specified in (6.13). There exists $K>0$ such that for all $n$,

$$
\left|C_{n}-c\left(t_{n+1}\right)\right| \leq K\left(\left|A_{n+1}-a\left(t_{n+1}\right)\right|+\left|B_{n+1}-b\left(t_{n+1}\right)\right|+\epsilon\right) .
$$

Proof. Using Lemma B.1, we have

Copyright $\odot$ by SIAM. Unauthorized reproduction of this article is prohibited. 


$$
\begin{aligned}
& \left|C_{n}-c\left(t_{n+1}\right)\right| \\
& \leq \mid G_{t_{n}}\left(\frac{A_{n+1}-\gamma_{n} r_{n}\left(B_{n+1}-A_{n+1}^{2}\right)}{\alpha_{n}^{2}\left(B_{n+1}-A_{n+1}^{2}\right)+\sigma_{n}^{2} B_{n+1}} \frac{\alpha_{n}}{\gamma_{n}}\right) \\
& -G_{t_{n+1}}\left[\frac{\alpha_{t_{n+1}}}{\gamma_{t_{n+1}} \sigma_{t_{n+1}}^{2}} \frac{a\left(t_{n+1}\right)+\gamma_{t_{n+1}}\left[a\left(t_{n+1}\right)^{2}-b\left(t_{n+1}\right)\right]}{b\left(t_{n+1}\right)}\right] \mid \\
& \leq K \epsilon+\left|\frac{A_{n+1}-\gamma_{n} r_{n}\left(B_{n+1}-A_{n+1}^{2}\right)}{\alpha_{n}^{2}\left(B_{n+1}-A_{n+1}^{2}\right)+\sigma_{n}^{2} B_{n+1}} \frac{\alpha_{n}}{\gamma_{n}}-\frac{\alpha_{t_{n+1}}}{\gamma_{t_{n+1}} \sigma_{t_{n+1}}^{2}} \frac{a\left(t_{n+1}\right)+\gamma_{t_{n+1}}\left[a\left(t_{n+1}\right)^{2}-b\left(t_{n+1}\right)\right]}{b\left(t_{n+1}\right)}\right| \\
& \leq K \epsilon+\left|\frac{A_{n+1}-\gamma_{n} r_{n}\left(B_{n+1}-A_{n+1}^{2}\right)}{\alpha_{n}^{2}\left(B_{n+1}-A_{n+1}^{2}\right)+\sigma_{n}^{2} B_{n+1}} \frac{\alpha_{n}}{\gamma_{n}}-\frac{\alpha_{n}}{\gamma_{n} \sigma_{n}^{2}} \frac{A_{n+1}-\gamma_{n} r_{n}\left(B_{n+1}-A_{n+1}^{2}\right)}{B_{n+1}}\right| \\
& +\left|\frac{\alpha_{n}}{\gamma_{n} \sigma_{n}^{2}} \frac{A_{n+1}-\gamma_{n} r_{n}\left(B_{n+1}-A_{n+1}^{2}\right)}{B_{n+1}}-\frac{\alpha_{t_{n+1}}}{\gamma_{t_{n+1}} \sigma_{t_{n+1}}^{2}} \frac{A_{n+1}-\gamma_{n} r_{n}\left(B_{n+1}-A_{n+1}^{2}\right)}{B_{n+1}}\right| \\
& +\mid \frac{\alpha_{t_{n+1}}}{\gamma_{t_{n+1}} \sigma_{t_{n+1}}^{2}} \frac{A_{n+1}-\gamma_{n} r_{n}\left(B_{n+1}-A_{n+1}^{2}\right)}{B_{n+1}} \\
& -\frac{\alpha_{t_{n+1}}}{\gamma_{t_{n+1}} \sigma_{t_{n+1}}^{2}} \frac{a\left(t_{n+1}\right)+\gamma_{t_{n+1}}\left[a\left(t_{n+1}\right)^{2}-b\left(t_{n+1}\right)\right]}{B_{n+1}} \mid \\
& +\mid \frac{\alpha_{t_{n+1}}}{\gamma_{t_{n+1}} \sigma_{t_{n+1}}^{2}} \frac{a\left(t_{n+1}\right)+\gamma_{t_{n+1}}\left[a\left(t_{n+1}\right)^{2}-b\left(t_{n+1}\right)\right]}{B_{n+1}} \\
& -\frac{\alpha_{t_{n+1}}}{\gamma_{t_{n+1}} \sigma_{t_{n+1}}^{2}} \frac{a\left(t_{n+1}\right)+\gamma_{t_{n+1}}\left[a\left(t_{n+1}\right)^{2}-b\left(t_{n+1}\right)\right]}{b\left(t_{n+1}\right)} \mid \\
& \leq K \epsilon+\left|\frac{1}{\frac{\alpha_{n}^{2}}{\sigma_{n}^{2}}\left(B_{n+1}-A_{n+1}^{2}\right)+B_{n+1}}-\frac{1}{B_{n+1}}\right|\left|\frac{\alpha_{n}}{\gamma_{n} \sigma_{n}^{2}}\left(A_{n+1}-\gamma_{n} r_{n}\left(B_{n+1}-A_{n+1}^{2}\right)\right)\right| \\
& +\left|\frac{\alpha_{n}}{\gamma_{n} \sigma_{n}^{2}}-\frac{\alpha_{t_{n+1}}}{\gamma_{t_{n+1}} \sigma_{t_{n+1}}^{2}}\right|\left|\frac{A_{n+1}-\gamma_{n} r_{n}\left(B_{n+1}-A_{n+1}^{2}\right)}{B_{n+1}}\right| \\
& +\left|\gamma_{n} r_{n} A_{n+1}^{2}-\gamma_{t_{n+1}} a\left(t_{n+1}\right)^{2}-\gamma_{n} r_{n} B_{n+1}+\gamma_{t_{n+1}} b\left(t_{n+1}\right)+A_{n+1}-a\left(t_{n+1}\right)\right|\left|\frac{\alpha_{t_{n+1}}}{\gamma_{t_{n+1}} \sigma_{t_{n+1}}^{2} B_{n+1}}\right| \\
& +\left|\frac{1}{B_{n+1}}-\frac{1}{b\left(t_{n+1}\right)}\right|\left|\frac{\alpha_{t_{n+1}}}{\gamma_{t_{n+1}} \sigma_{t_{n+1}}^{2}}\left\{a\left(t_{n+1}\right)+\gamma_{t_{n+1}}\left[a\left(t_{n+1}\right)^{2}-b\left(t_{n+1}\right)\right]\right\}\right| \\
& \text { (B.1) } \leq K \epsilon+\frac{\alpha}{\underline{\gamma} \underline{\sigma}^{2}}\left(K_{2}+\bar{\gamma} r\left(K_{2}+K_{2}^{2}\right)\right)\left|\frac{1}{\frac{\alpha_{n}^{2}}{\sigma_{n}^{2}}\left(B_{n+1}-A_{n+1}^{2}\right)+B_{n+1}}-\frac{1}{B_{n+1}}\right| \\
& +\frac{K_{2}+\bar{\gamma} r\left(K_{2}+K_{2}^{2}\right)}{k_{2}}\left|\frac{\alpha_{n}}{\gamma_{n} \sigma_{n}^{2}}-\frac{\alpha_{t_{n+1}}}{\gamma_{t_{n+1}} \sigma_{t_{n+1}}^{2}}\right| \\
& +\frac{\alpha}{\underline{\gamma} \underline{\sigma}^{2} k_{2}}\left|A_{n+1}-a\left(t_{n+1}\right)+\gamma_{n} r_{n} A_{n+1}^{2}-\gamma_{t_{n+1}} a\left(t_{n+1}\right)^{2}-\gamma_{n} r_{n} B_{n+1}+\gamma_{t_{n+1}} b\left(t_{n+1}\right)\right| \\
& +\frac{\alpha}{\underline{\gamma}^{2}}\left(K_{1}+\bar{\gamma}\left[K_{1}+K_{1}^{2}\right]\right)\left|\frac{1}{B_{n+1}}-\frac{1}{b\left(t_{n+1}\right)}\right| .
\end{aligned}
$$

Copyright (C) by SIAM. Unauthorized reproduction of this article is prohibited. 
We next study each summand on the right-hand side of the last inequality term by term. We consider (B.1) first:

$$
\begin{aligned}
& \left|\frac{1}{\frac{\alpha_{n}^{2}}{\sigma_{n}^{2}}\left(B_{n+1}-A_{n+1}^{2}\right)+B_{n+1}}-\frac{1}{B_{n+1}}\right| \\
& \leq\left|\frac{1}{\frac{\alpha_{n}^{2}}{\sigma_{n}^{2}}\left(B_{n+1}-A_{n+1}^{2}\right)+B_{n+1}}\right|\left|\frac{1}{B_{n+1}}\right|\left|\frac{\alpha_{n}^{2}}{\sigma_{n}^{2}}\left(B_{n+1}-A_{n+1}^{2}\right)\right| \leq\left|\frac{\alpha_{n}^{2}}{\sigma_{n}^{2}}\right|\left|\frac{B_{n+1}-A_{n+1}^{2}}{B_{n+1}^{2}}\right| \\
& \leq\left|\frac{e^{\int_{t_{n}}^{t_{n+1}} 2 r_{s} d s}\left(e^{\int_{t_{n}}^{t_{n+1}} \alpha_{s} d s}-1\right)^{2}}{\left(e^{\int_{t_{n}}^{t_{n+1}} \sigma_{s}^{2} d s}-1\right) e^{2 \int_{t_{n}}^{t_{n}+1} \mu_{s} d s}}\right|\left(\frac{K_{2}+K_{2}^{2}}{k_{2}^{2}}\right) \leq K e^{2 \alpha \epsilon} \frac{\alpha^{2} \epsilon^{2} e^{2 \alpha \epsilon}}{\underline{\sigma}^{2} \epsilon} \leq K e^{4 \alpha T} \frac{\alpha^{2}}{\underline{\sigma}^{2}} \epsilon
\end{aligned}
$$

(B.5) $\leq K \epsilon$,

where the second inequality follows because $\frac{\alpha_{n}^{2}}{\sigma_{n}^{2}}\left(B_{n+1}-A_{n+1}^{2}\right)>0$. Then, we consider (B.2):

$$
\begin{aligned}
& \left|\frac{\alpha_{n}}{\gamma_{n} \sigma_{n}^{2}}-\frac{\alpha_{t_{n+1}}}{\gamma_{t_{n+1}} \sigma_{t_{n+1}}^{2}}\right|
\end{aligned}
$$

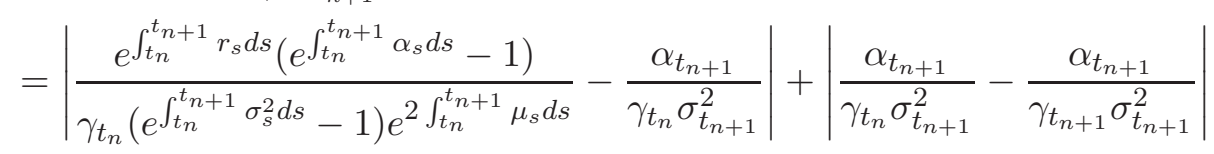

$$
\begin{aligned}
& =\frac{1}{\gamma_{t_{n}}}\left|\frac{e^{\int_{t_{n}}^{t_{n+1}} r_{s} d s} e^{\eta} \int_{t_{n}}^{t_{n+1}} \alpha_{s} d s}{e^{2 \int_{t_{n}}^{t_{n+1}} \mu_{s} d s} e^{\zeta} \int_{t_{n}}^{t_{n+1}} \sigma_{s}^{2} d s}-\frac{\alpha_{t_{n+1}}}{\sigma_{t_{n+1}}^{2}}\right|+\left|\frac{\alpha_{t_{n+1}}}{\sigma_{t_{n+1}}^{2}}\right|\left|\frac{1}{\gamma_{t_{n}}}-\frac{1}{\gamma_{t_{n+1}}}\right| \\
& (\mathrm{B} .6) \leq \frac{1}{\underline{\gamma}}\left|\frac{e^{\int_{t_{n}}^{t_{n+1}} r_{s} d s} e^{\eta}}{e^{\zeta} \cdot e^{2 \int_{t_{n}}^{t_{n+1}} \mu_{s} d s}}-1\right|\left|\frac{\int_{t_{n}}^{t_{n+1}} \alpha_{s} d s}{\int_{t_{n}}^{t_{n+1}} \sigma_{s}^{2} d s}\right|+\underline{\gamma}\left|\frac{\int_{t_{n}}^{t_{n+1}} \alpha_{s} d s}{\int_{t_{n}}^{t_{n+1}} \sigma_{s}^{2} d s}-\frac{\alpha_{t_{n+1}}}{\sigma_{t_{n+1}}^{2}}\right|+\frac{\alpha}{\underline{\gamma} \underline{\sigma}^{2}}\left|\gamma_{t_{n+1}}-\gamma_{t_{n}}\right|
\end{aligned}
$$

for some $\eta$ between 0 and $\int_{t_{n}}^{t_{n+1}} \alpha_{s} d s$ and $\zeta \in\left[0, \int_{t_{n}}^{t_{n+1}} \sigma_{s}^{2} d s\right]$ in light of the mean value theorem. Because

$$
e^{-\left[3(r+\alpha)+\bar{\sigma}^{2}\right] \epsilon} \leq \frac{e^{-r \epsilon} e^{-\alpha \epsilon}}{e^{2(r+\alpha) \epsilon} e^{\bar{\sigma}^{2} \epsilon}} \leq \frac{e^{\int_{t_{n}}^{t_{n}+1} r_{s} d s} e^{\eta}}{e^{2 \int_{t_{n}}^{t_{n}+1} \mu_{s} d s} e^{\zeta}} \leq \frac{e^{r \epsilon} e^{\alpha \epsilon}}{e^{-2(r+\alpha) \epsilon}} \leq e^{3(r+\alpha) \epsilon}
$$

we have

$$
\left|\frac{e^{\int_{t_{n}+1}^{t_{n+1}} r_{s} d s} e^{\eta}}{e^{2 \int_{t_{n}}^{t_{n+1}} \mu_{s} d s} e^{\zeta}}-1\right| \leq K \epsilon
$$

Moreover, since $\alpha_{t}$ and $\sigma_{t}$ are Lipschitz continuous, there exists $K>0$ (note that $\sigma_{s}^{2}$ is bounded below by $\underline{\sigma}^{2}$ ) such that

$$
\begin{aligned}
\alpha_{t_{n+1}}-K \epsilon & \leq \alpha_{s} \leq \alpha_{t_{n+1}}+K \epsilon, \\
\sigma_{t_{n+1}}^{2}-K \epsilon & \leq \sigma_{s}^{2} \leq \sigma_{t_{n+1}}^{2}+K \epsilon
\end{aligned}
$$

Copyright (C) by SIAM. Unauthorized reproduction of this article is prohibited. 
for all $s \in\left[t_{n}, t_{n+1}\right]$. Note that $\sigma_{t_{n+1}}^{2}-K \epsilon>\frac{1}{2} \underline{\sigma}^{2}$ for all $n$ (provided that $\epsilon$ is small enough), and

$$
\begin{gathered}
\frac{\alpha_{t_{n+1}}-K \epsilon}{\sigma_{t_{n+1}}^{2}+K \epsilon} \leq \frac{\int_{t_{n}}^{t_{n+1}} \alpha_{s} d s}{\int_{t_{n}}^{t_{n+1}} \sigma_{s}^{2} d s} \leq \frac{\alpha_{t_{n+1}}+K \epsilon}{\sigma_{t_{n+1}}^{2}-K \epsilon} \\
\frac{\alpha_{t_{n+1}}}{\sigma_{t_{n+1}}^{2}}-\left[\frac{\alpha_{t_{n+1}} K+\sigma_{t_{n+1}}^{2} K}{\sigma_{t_{n+1}}^{2}\left(\sigma_{t_{n+1}}^{2}+K \epsilon\right)}\right] \epsilon \leq \frac{\int_{t_{n}}^{t_{n+1}} \alpha_{s} d s}{\int_{t_{n}}^{t_{n+1}} \sigma_{s}^{2} d s} \leq \frac{\alpha_{t_{n+1}}}{\sigma_{t_{n+1}}^{2}}+\left[\frac{\alpha_{t_{n+1}} K+\sigma_{t_{n+1}}^{2} K}{\sigma_{t_{n+1}}^{2}\left(\sigma_{t_{n+1}}^{2}-K \epsilon\right)}\right] \epsilon, \\
\frac{\alpha_{t_{n+1}}}{\sigma_{t_{n+1}}^{2}}-\left[\frac{\alpha K+\bar{\sigma}^{2} K}{\underline{\sigma}^{4}}\right] \epsilon \leq \frac{\int_{t_{n}}^{t_{n+1}} \alpha_{s} d s}{\int_{t_{n}}^{t_{n+1}} \sigma_{s}^{2} d s} \leq \frac{\alpha_{t_{n+1}}}{\sigma_{t_{n+1}}^{2}}+2\left[\frac{\alpha K+\bar{\sigma}^{2} K}{\underline{\sigma}^{4}}\right] \epsilon .
\end{gathered}
$$

Using (B.7) and (B.8), (B.6) becomes

$$
\begin{aligned}
& \left|\frac{\alpha_{n}}{\gamma_{n} \sigma_{n}^{2}}-\frac{\alpha_{t_{n+1}}}{\gamma_{t_{n+1}} \sigma_{t_{n+1}}^{2}}\right| \\
\leq & \frac{1}{\underline{\underline{y}}}\left|\frac{e^{\int_{t_{n}}^{t_{n+1}} r_{s} d s} e^{\eta}}{e^{\zeta} \cdot e^{2 \int_{t_{n}}^{t_{n+1}} \mu_{s} d s}}-1\right|\left|\frac{\int_{t_{n}}^{t_{n+1}} \alpha_{s} d s}{\int_{t_{n}}^{t_{n+1}} \sigma_{s}^{2} d s}\right|+\underline{\gamma}\left|\frac{\int_{t_{n}}^{t_{n+1}} \alpha_{s} d s}{\int_{t_{n}}^{t_{n+1}} \sigma_{s}^{2} d s}-\frac{\alpha_{t_{n+1}}}{\sigma_{t_{n+1}}^{2}}\right|+\frac{\alpha}{\underline{\underline{\sigma^{2}}}}\left|\gamma_{t_{n+1}}-\gamma_{t_{n}}\right| \\
(\text { B.9) } \leq & K \epsilon\left(\frac{\int_{t_{n}}^{t_{n+1}} \alpha d s}{\int_{t_{n}}^{t_{n+1}} \underline{\sigma}^{2} d s}\right)+K \epsilon+K \epsilon \leq K^{\prime} \epsilon .
\end{aligned}
$$

Furthermore, for the term (B.3), by using Lemma B.1,

$$
\begin{aligned}
& \left|\gamma_{n} r_{n} A_{n+1}^{2}-\gamma_{t_{n+1}} a\left(t_{n+1}\right)^{2}\right| \\
\leq & \gamma_{n}\left(r_{n}-1\right) A_{n+1}^{2}+\gamma_{n}\left|A_{n+1}-a\left(t_{n+1}\right)\right|\left|A_{n+1}+a\left(t_{n+1}\right)\right|+a\left(t_{n+1}\right)^{2}\left|\gamma_{n}-\gamma_{t_{n+1}}\right| \\
\leq & \bar{\gamma}\left(e^{r \epsilon}-1\right) K_{2}^{2}+\bar{\gamma}\left|A_{n+1}-a\left(t_{n+1}\right)\right|\left(K_{1}+K_{2}\right)+K_{2}^{2} K \epsilon \\
\leq & K\left(\epsilon+\left|A_{n+1}-a\left(t_{n+1}\right)\right|\right), \\
& \left|\gamma_{n} r_{n} B_{n+1}-\gamma_{t_{n+1}} b\left(t_{n+1}\right)\right| \\
\leq & \gamma_{n}\left(e^{r \epsilon}-1\right) B_{n+1}+\gamma_{n}\left|B_{n+1}-b\left(t_{n+1}\right)\right|+b\left(t_{n+1}\right)\left|\gamma_{n}-\gamma_{t_{n+1}}\right| \\
\leq & K\left(\epsilon+\left|B_{n+1}-b\left(t_{n+1}\right)\right|\right) .
\end{aligned}
$$

Therefore, we further have

$$
\begin{aligned}
& \left|A_{n+1}-a\left(t_{n+1}\right)+\gamma_{n} r_{n} A_{n+1}^{2}-\gamma_{t_{n+1}} a\left(t_{n+1}\right)^{2}-\gamma_{n} r_{n} B_{n+1}+\gamma_{t_{n+1}} b\left(t_{n+1}\right)\right| \\
\leq & K\left(\epsilon+\left|A_{n+1}-a\left(t_{n+1}\right)\right|+\left|B_{n+1}-b\left(t_{n+1}\right)\right|\right) .
\end{aligned}
$$

Finally, for the term (B.4), by using Lemma B.1, we have

$$
\left|\frac{1}{B_{n+1}}-\frac{1}{b\left(t_{n+1}\right)}\right| \leq\left|\frac{B_{n+1}-b\left(t_{n+1}\right)}{B_{n+1} b\left(t_{n+1}\right)}\right| \leq\left|\frac{B_{n+1}-b\left(t_{n+1}\right)}{k_{2} k_{1}}\right| \leq K\left|B_{n+1}-b\left(t_{n+1}\right)\right| .
$$

Copyright (C) by SIAM. Unauthorized reproduction of this article is prohibited. 
We now put (B.5), (B.9), (B.10), and (B.11) into (B.1)-(B.4), and we prove Lemma B.3:

$$
\begin{aligned}
& \left|C_{n}-c\left(t_{n+1}\right)\right| \\
\leq & K \epsilon+\frac{\alpha}{\underline{\gamma} \underline{\sigma}^{2}}\left(K_{2}+\bar{\gamma} r\left(K_{2}+K_{2}^{2}\right)\right)\left|\frac{1}{\frac{\alpha_{n}^{2}}{\sigma_{n}^{2}}\left(B_{n+1}-A_{n+1}^{2}\right)+B_{n+1}}-\frac{1}{B_{n+1}}\right| \\
& +\frac{K_{2}+\bar{\gamma} r\left(K_{2}+K_{2}^{2}\right)}{k_{2}}\left|\frac{\alpha_{n}}{\gamma_{n} \sigma_{n}^{2}}-\frac{\alpha_{t_{n+1}}}{\gamma_{t_{n+1}} \sigma_{t_{n+1}}^{2}}\right| \\
& +\frac{\alpha}{\underline{\gamma} \underline{\sigma}^{2} k_{2}}\left|A_{n+1}-a\left(t_{n+1}\right)+\gamma_{n} r_{n} A_{n+1}^{2}-\gamma_{t_{n+1}} a\left(t_{n+1}\right)^{2}-\gamma_{n} r_{n} B_{n+1}+\gamma_{t_{n+1}} b\left(t_{n+1}\right)\right| \\
& +\frac{\alpha}{\underline{\gamma} \underline{\sigma}^{2}}\left(K_{1}+\bar{\gamma}\left[K_{1}+K_{1}^{2}\right]\right)\left|\frac{1}{B_{n+1}}-\frac{1}{b\left(t_{n+1}\right)}\right| \\
\leq & K \epsilon+K \epsilon+K \epsilon+K\left(\epsilon+\left|A_{n+1}-a\left(t_{n+1}\right)\right|+\left|B_{n+1}-b\left(t_{n+1}\right)\right|\right)+K\left|B_{n+1}-b\left(t_{n+1}\right)\right| \\
\leq & K^{\prime}\left(\left|A_{n+1}-a\left(t_{n+1}\right)\right|+\left|B_{n+1}-b\left(t_{n+1}\right)\right|+\epsilon\right) .
\end{aligned}
$$

Lemma B.4. Fix $\epsilon>0 . a(t)$ and $b(t)$ are defined as in (6.14), and $c(t)$ satisfies (6.6). Then there exists $K>0$ such that for all $n$,

$$
\begin{aligned}
\left|a\left(t_{n}\right)-a\left(t_{n+1}\right)+\epsilon a^{\prime}\left(t_{n+1}\right)\right| & \leq K \epsilon^{2}, \\
\left|b\left(t_{n}\right)-b\left(t_{n+1}\right)+\epsilon b^{\prime}\left(t_{n+1}\right)\right| & \leq K \epsilon^{2} .
\end{aligned}
$$

Proof. By the mean value theorem, there is an $\eta \in\left[t_{n}, t_{n+1}\right]$,

$$
\left|a\left(t_{n}\right)-a\left(t_{n+1}\right)+\epsilon a^{\prime}\left(t_{n+1}\right)\right| \leq \epsilon\left|a^{\prime}\left(t_{n+1}\right)-a^{\prime}(\eta)\right| .
$$

By (6.15), we have

$$
\begin{aligned}
& \left|a^{\prime}\left(t_{n+1}\right)-a^{\prime}(\eta)\right| \\
\leq & \left|\left(r_{\eta}+\alpha_{\eta} c(\eta)\right) a(\eta)-\left(r_{t_{n+1}}+\alpha_{t_{n+1}} c\left(t_{n+1}\right)\right) a\left(t_{n+1}\right)\right| \\
\leq & \left|r_{\eta}+\alpha_{\eta} c(\eta)\right|\left|a(\eta)-a\left(t_{n+1}\right)\right|+\left|a\left(t_{n+1}\right)\right|\left|\left(r_{\eta}+\alpha_{\eta} c(\eta)\right)-\left(r_{t_{n+1}}+\alpha_{t_{n+1}} c\left(t_{n+1}\right)\right)\right| \\
\leq & (r+\alpha)\left(t_{n+1}-\eta\right)\left|a^{\prime}(\zeta)\right|+K_{1}\left(\left|r_{\eta}-r_{t_{n+1}}\right|+\left|\alpha_{\eta}-\alpha_{t_{n+1}}\right| c(\eta)+\left|\alpha_{t_{n+1}}\right|\left|c(\eta)-c\left(t_{n+1}\right)\right|\right) \\
\leq & K \epsilon\left|\left(r_{\zeta}+\alpha_{\zeta} c(\zeta)\right) a(\zeta)\right|+K_{1}(K \epsilon+K \epsilon+\alpha K \epsilon) \leq K \epsilon,
\end{aligned}
$$

where the mean value theorem is applied in the third inequality for some $\zeta \in\left[\eta, t_{n+1}\right]$, and the fourth inequality follows because of Lipschitz continuity of $r_{t}$ and $\alpha_{t}$, boundedness of $c(t)$, and Lipschitz continuity of $c(t)$ from Lemma B.2. And hence (B.12) follows. Similarly, for (B.13), by the mean value theorem, there is an $\eta^{\prime} \in\left[t_{n}, t_{n+1}\right]$,

$$
\left|b\left(t_{n}\right)-b\left(t_{n+1}\right)+\epsilon b^{\prime}\left(t_{n+1}\right)\right| \leq \epsilon\left|b^{\prime}\left(t_{n+1}\right)-b^{\prime}\left(\eta^{\prime}\right)\right| .
$$

Copyright (C) by SIAM. Unauthorized reproduction of this article is prohibited. 
By (6.17), we have

$$
\begin{aligned}
& \left|b^{\prime}\left(t_{n+1}\right)-b^{\prime}\left(\eta^{\prime}\right)\right| \\
\leq & \left|\left(2 r_{\eta^{\prime}}+2 \alpha_{\eta^{\prime}} c\left(\eta^{\prime}\right)+\sigma_{\eta^{\prime}}^{2} c\left(\eta^{\prime}\right)^{2}\right) b\left(\eta^{\prime}\right)-\left(2 r_{t_{n+1}}+2 \alpha_{t_{n+1}} c\left(t_{n+1}\right)+\sigma_{t_{n+1}}^{2} c\left(t_{n+1}\right)^{2}\right) b\left(t_{n+1}\right)\right| \\
\leq & \left|2 r_{\eta^{\prime}}+2 \alpha_{\eta^{\prime}} c\left(\eta^{\prime}\right)+\sigma_{\eta^{\prime}}^{2} c\left(\eta^{\prime}\right)^{2}\right|\left|b\left(\eta^{\prime}\right)-b\left(t_{n+1}\right)\right| \\
& +\left|b\left(t_{n+1}\right)\right|\left(2\left|r_{\eta^{\prime}}-r_{t_{n+1}}\right|+2\left|\alpha_{\eta^{\prime}}-\alpha_{t_{n+1}}\right| c\left(\eta^{\prime}\right)+2\left|\alpha_{t_{n+1}}\right|\left|c\left(\eta^{\prime}\right)-c\left(t_{n+1}\right)\right|\right) \\
& +\left|b\left(t_{n+1}\right)\right|\left(\left|\sigma_{\eta^{\prime}}^{2}-\sigma_{t_{n+1}}^{2}\right| c\left(\eta^{\prime}\right)+\left|\sigma_{t_{n+1}}^{2}\right|\left|c\left(\eta^{\prime}\right)-c\left(t_{n+1}\right)\right|\right) \\
\leq & \left(2 r+2 \alpha+\bar{\sigma}^{2}\right)\left(t_{n+1}-\eta^{\prime}\right)\left|b^{\prime}\left(\zeta^{\prime}\right)\right|+K_{2}\left(2 K \epsilon+2 K \epsilon+2 \alpha K \epsilon+K \epsilon+\bar{\sigma}^{2} K \epsilon\right) \\
\leq & K \epsilon\left|\left(2 r_{\zeta^{\prime}}+2 \alpha_{\zeta^{\prime}} c\left(\zeta^{\prime}\right)+\sigma_{\zeta^{\prime}}^{2} c\left(\zeta^{\prime}\right)^{2}\right) b\left(\zeta^{\prime}\right)\right|+K \epsilon \leq K \epsilon .
\end{aligned}
$$

As a consequence, the inequality (B.13) also follows.

Theorem B.5. Fix $\epsilon>0 . a(t)$ and $b(t)$ are defined as in (6.14), $c(t)$ satisfies (6.6), and $A_{n}, B_{n}$, and $C_{n}$ are given in Theorem 4.1 with coefficients as specified in (6.13). There exists $K>0$ such that $\left|A_{n}-a\left(t_{n}\right)\right| \leq K \epsilon$ and $\left|B_{n}-b\left(t_{n}\right)\right| \leq K \epsilon$.

Proof. Denote $u_{n}:=\left|A_{n}-a\left(t_{n}\right)\right|$ and $v_{n}:=\left|B_{n}-b\left(t_{n}\right)\right|$. By Lemmas B.3 and B.4, (4.4), (6.15), uniformly boundedness of the parameters, and boundedness results in Lemma B.1, we have

$$
\begin{aligned}
& u_{n} \leq\left|\left(r_{n}+\alpha_{n} C_{n}\right) A_{n+1}-a\left(t_{n+1}\right)+\epsilon a^{\prime}\left(t_{n+1}\right)\right|+K \epsilon^{2} \\
& \leq \mid\left(e^{\int_{t_{n}}^{t_{n+1}} r_{s} d s}+e^{\int_{t_{n}}^{t_{n+1}} r_{s} d s}\left(e^{\int_{t_{n}}^{t_{n+1}} \alpha_{s} d s}-1\right) C_{n}\right) A_{n+1}-a\left(t_{n+1}\right) \\
& -\epsilon\left(r_{t_{n+1}}+\alpha_{t_{n+1}} c\left(t_{n+1}\right)\right) a\left(t_{n+1}\right) \mid+K \epsilon^{2} \\
& \leq\left|e^{\int_{t_{n}}^{t_{n+1}} r_{s} d s} A_{n+1}-\left(1+\epsilon r_{t_{n+1}}\right) a\left(t_{n+1}\right)\right|+\left|e^{\int_{t_{n}}^{t_{n+1}} r_{s} d s}\left(e^{\int_{t_{n}}^{t_{n+1}} \alpha_{s} d s}-1\right)-\epsilon \alpha_{t_{n+1}}\right|\left|C_{n} A_{n+1}\right| \\
& +\epsilon\left|\alpha_{t_{n+1}} A_{n+1}\right|\left|C_{n}-c\left(t_{n+1}\right)\right|+\epsilon\left|\alpha_{t_{n+1}} c\left(t_{n+1}\right)\right|\left|A_{n+1}-a\left(t_{n+1}\right)\right|+K \epsilon^{2} \\
& \leq\left(1+r_{t_{n+1}} \epsilon\right) u_{n+1}+\left|A_{n+1}\right|\left|e^{\int_{t_{n}}^{t_{n+1}} r_{s} d s}-1-r_{t_{n+1}} \epsilon\right| \\
& +\left|A_{n+1}\right|\left(\left|\left(e^{\int_{t_{n}}^{t_{n+1}} r_{s} d s}-1\right)\left(e^{\int_{t_{n}}^{t_{n+1}} \alpha_{s} d s}-1\right)\right|+\left|e^{\int_{t_{n}}^{t_{n+1}} \alpha_{s} d s}-1-\epsilon \alpha_{t_{n+1}}\right|\right) \\
& +\left|\alpha_{t_{n+1}} A_{n+1}\right| \epsilon\left(u_{n+1}+v_{n+1}+\epsilon\right)+\left|\alpha_{t_{n+1}}\right| \epsilon u_{n+1}+K \epsilon^{2} .
\end{aligned}
$$

By Taylor's theorem, we have

$$
\begin{aligned}
\left|e^{\int_{t_{n}}^{t_{n+1}} r_{s} d s}-1-r_{t_{n+1}} \epsilon\right| & \leq\left|\int_{t_{n}}^{t_{n+1}} r_{s} d s-\int_{t_{n}}^{t_{n+1}} r_{t_{n+1}} d s+\frac{1}{2}\left(\int_{t_{n}}^{t_{n+1}} r_{s} d s\right)^{2} e^{\eta}\right| \\
& \leq \int_{t_{n}}^{t_{n+1}}\left|r_{s}-r_{t_{n+1}}\right| d s+\frac{1}{2} e^{r \epsilon}\left(\int_{t_{n}}^{t_{n+1}}\left|r_{s}\right| d s\right)^{2} \\
& \leq \int_{t_{n}}^{t_{n+1}} K \epsilon d s+\frac{1}{2} e^{r T}\left(\int_{t_{n}}^{t_{n+1}} r d s\right)^{2} \leq K \epsilon^{2}
\end{aligned}
$$

Copyright (C) by SIAM. Unauthorized reproduction of this article is prohibited. 
where $\eta$ is some number between 0 and $\int_{t_{n}}^{t_{n+1}} r_{s} d s$, and the third inequality follows because $r_{t}$ is Lipschitz continuous and bounded above by $r$. Similarly, we have

$$
\left|e^{\int_{t_{n}}^{t_{n+1}} \alpha_{s} d s}-1-\alpha_{t_{n+1}} \epsilon\right| \leq K \epsilon^{2} .
$$

By the mean value theorem, we also have

$$
\left|e^{\int_{t_{n}}^{t_{n+1}} r_{s} d s}-1\right| \leq\left|e^{\eta^{\prime}} \int_{t_{n}}^{t_{n+1}} r_{s} d s\right| \leq\left(\int_{t_{n}}^{t_{n+1}} r d s\right) e^{r \epsilon} \leq r \epsilon e^{r T} \leq K \epsilon
$$

for an $\eta^{\prime}$ being some number between 0 and $\int_{t_{n}}^{t_{n+1}} r_{s} d s$. Similarly, we have

$$
\left|e^{\int_{t_{n}}^{t_{n+1}} \alpha_{s} d s}-1\right| \leq K \epsilon
$$

Combining all these results, (B.14) becomes

$$
\begin{aligned}
& u_{n} \leq(1+r \epsilon) u_{n+1}+K_{2}\left|e^{\int_{t_{n}}^{t_{n+1}} r_{s} d s}-1-r_{t_{n+1}} \epsilon\right| \\
&+K_{2}\left(\left|\left(e^{\int_{t_{n}+1}^{t_{n}}} r_{s} d s-1\right)\left(e^{\int_{t_{n}+1}^{t_{n}} \alpha_{s} d s}-1\right)\right|+\left|e^{\int_{t_{n}}^{t_{n+1}} \alpha_{s} d s}-1-\epsilon \alpha_{t_{n+1}}\right|\right) \\
&+\alpha K_{2} \epsilon\left(u_{n+1}+v_{n+1}+\epsilon\right)+\alpha \epsilon u_{n+1}+K \epsilon^{2} \\
& \leq(1+M \epsilon) u_{n+1}+K_{2} K \epsilon^{2}+K\left(K \epsilon^{2}+K \epsilon^{2}\right)+M \epsilon\left(u_{n+1}+v_{n+1}\right)+K \epsilon^{2}+M \epsilon u_{n+1}+K \epsilon^{2} \\
&\left(\text { B.17) } \quad \leq u_{n+1}+\epsilon M\left(u_{n+1}+v_{n+1}\right)+K \epsilon^{2} .\right.
\end{aligned}
$$

Next, for $v_{n}$, using Lemma B.4, (4.6), and (6.17), we have

$$
\begin{aligned}
& v_{n} \leq\left|\left[\left(r_{n}+\alpha_{n} C_{n}\right)^{2}+\sigma_{n}^{2} C_{n}^{2}\right] B_{n+1}-b\left(t_{n+1}\right)+\epsilon b^{\prime}\left(t_{n+1}\right)\right|+K \epsilon^{2} \\
& \leq \mid\left[\left(e^{\int_{t_{n}}^{t_{n}+1} r_{s} d s}+e^{\int_{t_{n}}^{t_{n+1}}} r_{s} d s\left(e^{\int_{t_{n}}^{t_{n+1}} \alpha_{s} d s}-1\right) C_{n}\right)^{2}+\left(e^{\int_{t_{n}}^{t_{n+1}} \sigma_{s}^{2} d s}-1\right) e^{2 \int_{t_{n}}^{t_{n+1}} \mu_{s} d s} C_{n}^{2}\right] B_{n+1} \\
& -b\left(t_{n+1}\right)-\epsilon\left[2\left(r_{t_{n+1}}+\alpha_{t_{n+1}} c\left(t_{n+1}\right)\right)+\sigma_{t_{n+1}}^{2} c\left(t_{n+1}\right)^{2}\right] b\left(t_{n+1}\right) \mid+K \epsilon^{2} \\
& \leq\left|e^{\int_{t_{n}}^{t_{n+1}} 2 r_{s} d s} B_{n+1}-\left(1+2 \epsilon r_{t_{n+1}}\right) b\left(t_{n+1}\right)\right| \\
& +2\left|e^{\int_{t_{n}}^{t_{n+1}} 2 r_{s} d s}\left(e^{\int_{t_{n}}^{t_{n+1}} \alpha_{s} d s}-1\right) C_{n} B_{n+1}-\epsilon \alpha_{t_{n+1}} c\left(t_{n+1}\right) b\left(t_{n+1}\right)\right| \\
& +\left|e^{\int_{t_{n}}^{t_{n}} 2 r_{s} d s}\left(e^{\int_{t_{n}}^{t_{n+1}} \alpha_{s} d s}-1\right)^{2} C_{n}^{2} B_{n+1}\right| \\
& +\left|\left(e^{\int_{t_{n}}^{t_{n+1}} \sigma_{s}^{2} d s}-1\right) e^{2 \int_{t_{n}}^{t_{n+1}} \mu_{s} d s} C_{n}^{2} B_{n+1}-\epsilon \sigma_{t_{n+1}}^{2} c\left(t_{n+1}\right)^{2} b\left(t_{n+1}\right)\right|+K \epsilon^{2} \\
& \leq\left|e^{\int_{t_{n}}^{t_{n+1}} 2 r_{s} d s}-1-2 \epsilon r_{t_{n+1}}\right|\left|B_{n+1}\right|+\left|1+2 \epsilon r_{t_{n+1}}\right|\left|B_{n+1}-b\left(t_{n+1}\right)\right| \\
& +2\left|e^{\int_{t_{n}}^{t_{n+1}} \alpha_{s} d s}-1-\epsilon \alpha_{t_{n+1}}\right|\left|C_{n} B_{n+1}\right|+2 \epsilon\left|\alpha_{t_{n+1}} B_{n+1}\right|\left|C_{n}-c\left(t_{n+1}\right)\right| \\
& +2 \epsilon\left|\alpha_{t_{n+1}} c\left(t_{n+1}\right)\right|\left|B_{n+1}-b\left(t_{n+1}\right)\right| \\
& +2\left|\left(e^{\int_{t_{n}}^{t_{n+1}} 2 r_{s} d s}-1\right)\left(e^{\int_{t_{n}}^{t_{n+1}} \alpha_{s} d s}-1\right) C_{n} B_{n+1}\right|+\left|e^{\int_{t_{n}}^{t_{n+1}} 2 r_{s} d s}\left(e^{\int_{t_{n}}^{t_{n}} \alpha_{s} d s}-1\right)^{2} C_{n}^{2} B_{n+1}\right| \\
& +\left|\left(e^{\int_{t_{n}}^{t_{n}} \sigma_{s}^{2} d s}-1\right)\left(e^{2 \int_{t_{n}}^{t_{n+1}} \mu_{s} d s}-1\right)\right|\left|C_{n}^{2} B_{n+1}\right|+\left|e^{\int_{t_{n}}^{t_{n+1}} \sigma_{s}^{2} d s}-1-\epsilon \sigma_{t_{n+1}}^{2}\right| C_{n}^{2} B_{n+1} \mid \\
& +\epsilon \sigma_{t_{n+1}}^{2}\left|B_{n+1}\right|\left|C_{n}^{2}-c\left(t_{n+1}\right)^{2}\right|+\epsilon \sigma_{t_{n+1}}^{2} c\left(t_{n+1}\right)^{2}\left|B_{n+1}-b\left(t_{n+1}\right)\right|+K \epsilon^{2}
\end{aligned}
$$

Copyright (C by SIAM. Unauthorized reproduction of this article is prohibited. 


$$
\begin{aligned}
& \leq K_{2}\left|e^{\int_{t_{n}}^{t_{n}+1} 2 r_{s} d s}-1-2 \epsilon r_{t_{n+1}}\right|+(1+2 r \epsilon) v_{n+1}+2 K_{2}\left|e^{\int_{t_{n}}^{t_{n+1}} \alpha_{s} d s}-1-\epsilon \alpha_{t_{n+1}}\right| \\
&+2 \alpha K_{2} \epsilon\left|C_{n}-c\left(t_{n+1}\right)\right|+2 \epsilon \alpha v_{n+1}+2 K_{2}\left|\left(e^{\int_{t_{n}}^{t_{n+1}} 2 r_{s} d s}-1\right)\left(e^{\int_{t_{n}}^{t_{n+1}} \alpha_{s} d s}-1\right)\right| \\
&+K_{2} e^{2 r \epsilon}\left(e^{\int_{t_{n}}^{t_{n+1}} \alpha_{s} d s}-1\right)^{2}+K_{2}\left|\left(e^{\int_{t_{n}}^{t_{n+1}}} \sigma_{s}^{2} d s-1\right)\left(e^{2 \int_{t_{n}}^{t_{n+1}} \mu_{s} d s}-1\right)\right| \\
& \text { (B.18) } \quad+K_{2}\left|e^{\int_{t_{n}}^{t_{n+1}} \sigma_{s}^{2} d s}-1-\epsilon \sigma_{t_{n+1}}^{2}\right|+\epsilon K_{2} \bar{\sigma}^{2}\left|C_{n}^{2}-c\left(t_{n+1}\right)^{2}\right|+\epsilon \bar{\sigma}^{2} v_{n+1}+K \epsilon^{2} .
\end{aligned}
$$

Applying Taylor's theorem as for (B.15), we deduce that

$$
\begin{aligned}
& \left|e^{\int_{t_{n}}^{t_{n+1}} 2 r_{s} d s}-1-2 \epsilon r_{t_{n+1}}\right| \leq K \epsilon^{2}, \quad\left|e^{\int_{t_{n}}^{t_{n+1}} \alpha_{s} d s}-1-2 \epsilon \alpha_{t_{n+1}}\right| \leq K \epsilon^{2}, \\
& \left|e^{\int_{t_{n}}^{t_{n+1}} \sigma_{s}^{2} d s}-1-2 \epsilon \sigma_{t_{n+1}}^{2}\right| \leq K \epsilon^{2}
\end{aligned}
$$

Using the mean value theorem as for (B.16), we also obtain

$$
\begin{array}{ll}
\left|e^{\int_{t_{n}}^{t_{n+1}} 2 r_{s} d s}-1\right| \leq K \epsilon, & \left|e^{\int_{t_{n}}^{t_{n+1}} \alpha_{s} d s}-1\right| \leq K \epsilon, \\
\left|e^{\int_{t_{n}}^{t_{n+1}} 2 \mu_{s} d s}-1\right| \leq K \epsilon, & \left|e^{\int_{t_{n}}^{t_{n+1}} \sigma_{s}^{2} d s}-1\right| \leq K \epsilon .
\end{array}
$$

By Lemma B.3, we have

$$
\begin{aligned}
\left|C_{n}-C\left(t_{n+1}\right)\right| & \leq K\left(u_{n+1}+v_{n+1}\right)+K \epsilon \\
\left|C_{n}^{2}-C\left(t_{n+1}\right)^{2}\right| & =\left|C_{n}-C\left(t_{n+1}\right)\right|\left|C_{n}+C\left(t_{n+1}\right)\right| \leq 2 K\left(u_{n+1}+v_{n+1}\right)+2 K \epsilon .
\end{aligned}
$$

Combining these estimates, (B.18) becomes

$$
\begin{aligned}
v_{n} \leq & K_{2}\left|e^{\int_{t_{n}}^{t_{n+1}} 2 r_{s} d s}-1-2 \epsilon r_{t_{n+1}}\right|+(1+2 r \epsilon) v_{n+1}+2 K_{2}\left|e^{\int_{t_{n}}^{t_{n}} \alpha_{s} d s}-1-\epsilon \alpha_{t_{n+1}}\right| \\
& +2 \alpha K_{2} \epsilon\left|C_{n}-c\left(t_{n+1}\right)\right|+2 \epsilon \alpha v_{n+1}+2 K_{2}\left|\left(e^{\int_{t_{n}}^{t_{n+1}} 2 r_{s} d s}-1\right)\left(e^{\int_{t_{n}}^{t_{n+1}} \alpha_{s} d s}-1\right)\right| \\
& +K_{2} e^{2 r \epsilon}\left(e^{\int_{t_{n}}^{t_{n+1}} \alpha_{s} d s}-1\right)^{2}+K_{2}\left|\left(e^{\int_{t_{n}}^{t_{n+1}}} \sigma_{s}^{2} d s-1\right)\left(e^{2 \int_{t_{n}}^{t_{n+1}} \mu_{s} d s}-1\right)\right| \\
& +K_{2}\left|e^{\int_{t_{n}}^{t_{n+1}}} \sigma_{s}^{2} d s-1-\epsilon \sigma_{t_{n+1}}^{2}\right|+\epsilon K_{2} \bar{\sigma}^{2}\left|C_{n}^{2}-c\left(t_{n+1}\right)^{2}\right|+\epsilon \bar{\sigma}^{2} v_{n+1}+K \epsilon^{2} \\
\leq & K_{2} K \epsilon^{2}+\left[1+\left(2 \mu+\bar{\sigma}^{2}\right) \epsilon\right] v_{n+1}+2 K_{2} K \epsilon^{2}+2 \alpha K_{2} \epsilon\left(K\left(u_{n+1}+v_{n+1}\right)+K \epsilon\right)+2 K_{2} K^{2} \epsilon^{2} \\
& +K_{2} e^{2 r T} K^{2} \epsilon^{2}+K_{2} K^{2} \epsilon^{2}+K_{2} K \epsilon+\epsilon K_{2} \bar{\sigma}^{2}\left(2 K\left(u_{n+1}+v_{n+1}\right)+2 K \epsilon\right)+K \epsilon^{2} \\
(\text { B.19) } & \leq v_{n+1}+\epsilon M\left(u_{n+1}+v_{n+1}\right)+K \epsilon^{2} .
\end{aligned}
$$

Define $x_{n}:=u_{n}+v_{n}$. By (B.17), (B.19), and $u_{N}=v_{N}=0$, we have

$$
x_{n} \leq(1+\epsilon M) x_{n+1}+K \epsilon^{2} \leq \sum_{k=n}^{N-1}(1+\epsilon M)^{k-n} K \epsilon^{2} \leq K \epsilon^{2} \frac{(1+\epsilon M)^{N-n}-1}{\epsilon M} \leq K \epsilon \frac{e^{M T}-1}{M} \leq K \epsilon .
$$

Thus $\left|A_{n}-a\left(t_{n}\right)\right| \leq u_{n} \leq x_{n} \leq K \epsilon$. Similarly, we can show that $\left|B_{n}-b\left(t_{n}\right)\right| \leq K \epsilon$. 
Based on the previous lemmas and theorem, we can now deduce that the solutions in the discrete time model converge to that in the continuous time counterpart as the time interval goes to zero.

Proof of Theorem 6.4. This theorem follows by applying Lemmas B.2 and B.3 and Theorem B.5:

$$
\begin{aligned}
\left|C_{n}-c\left(t_{n}\right)\right| & \leq\left|c\left(t_{n+1}\right)-c\left(t_{n}\right)\right|+\left|C_{n}-c\left(t_{n+1}\right)\right| \\
& \leq K \epsilon+K\left(\left|A_{n+1}-a\left(t_{n+1}\right)\right|+\left|B_{n+1}-b\left(t_{n+1}\right)\right|+\epsilon\right) \\
& \leq K \epsilon .
\end{aligned}
$$

Acknowledgments. The authors express their sincere gratitude for the inspiring comments from the anonymous referees. The third author expresses his sincere gratitude for the hospitality of the Hausdorff Center for Mathematics of the University of Bonn during his fruitful stay in the Hausdorff Trimester Program "Stochastic Dynamics in Economics and Finance."

\section{REFERENCES}

[1] S. Basak And G. Chabakauri, Dynamic mean-variance asset allocation, Rev. Financ. Stud., 23 (2010), pp. 2970-3016.

[2] A. Bensoussan, K. C. Wong, And S. C. P. Yam, Mean-variance precommitment policies revisited via a mean-field technique, in Recent Advances in Financial Engineering 2012, World Scientific, Singapore, to appear.

[3] T. Buörk And A. Murgoci, A General Theory of Markovian Time Inconsistent Stochastic Control Problems, working paper, Stockholm School of Economics, Stockholm, Sweden, 2010.

[4] T. BJörk, A. Murgoci, And X. Y. Zhou, Mean-variance portfolio optimization with state-dependent risk aversion, Math. Finance, 24 (2014), pp. 1-24.

[5] P. CHEN AND H. YANG, Markowitz's mean-variance asset-liability management with regime switching: A multi-period model, Appl. Math. Finance, 18 (2011), pp. 29-50.

[6] P. Chen, H. YAng, And G. YIn, Markowitz's mean-variance asset-liability management with regime switching: A continuous-time model, Insurance Math. Econom., 43 (2008), pp. 456-465.

[7] J. Cvitanić and I. Karatzas, Convex duality in constrained portfolio optimization, Ann. Appl. Probab., 2 (1992), pp. 767-818.

[8] I. Ekeland AND A. LAZRAK, Being Serious about Non-commitment: Subgame Perfect Equilibrium in Continuous Time, preprint, University of British Columbia, Vancouver, Canada, 2006.

[9] I. Ekeland, O. Mbodji, and T. A. Pirvu, Time-consistent portfolio management, SIAM J. Financial Math., 3 (2012), pp. 1-32.

[10] I. Ekeland And T. A. Pirvu, Investment and consumption without commitment, Math. Financ. Econ., 2 (2008), pp. 57-86.

[11] P. A. Forsyth And J. WAng, Continuous time mean variance asset allocation: A time-consistent strategy, European J. Oper. Res., 209 (2011), pp. 184-201.

[12] H. He And H. F. PAGEs, Labor income, borrowing constraints, and equilibrium asset prices: A duality approach, Econom. Theory, 3 (1993), pp. 663-696.

[13] A. J. Heunis and C. LabBÉ, Convex duality in constrained mean-variance portfolio optimization, Adv. in Appl. Probab., 39 (2007), pp. 77-104.

[14] Y. Hu, H. Jin, AND X. Y. ZHou, Time-inconsistent stochastic linear-quadratic control, SIAM J. Control Optim., 50 (2012), pp. 1548-1572.

[15] Y. Hu AND X. Y. ZHOU, Constrained stochastic LQ control with random coefficients, and application to portfolio selection, SIAM J. Control Optim., 44 (2005), pp. 444-466.

[16] E. M. Kryger and M. Steffensen, Some Solvable Portfolio Problems with Quadratic and Collective Objectives, working paper, 2010.

Copyright $\odot$ by SIAM. Unauthorized reproduction of this article is prohibited. 
[17] D. Li And W. L. NG, Optimal dynamic portfolio selection: Multiperiod mean-variance formulation, Math. Finance, 10 (2000), pp. 387-406.

[18] H. Markowitz, Portfolio selection, J. Finance, 7 (1952), pp. 77-91.

[19] R. C. Merton, Optimum consumption and portfolio rules in a continuous-time model, J. Econom. Theory, 3 (1971), pp. 373-413.

[20] B. Peleg And M. E. YAari, On the existence of a consistent course of action when tastes are changing, Rev. Econom. Stud., 40 (1973), pp. 391-401.

[21] R. Pollak, Consistent planning, Rev. Econom. Stud., 35 (1968), pp. 201-208.

[22] P. A. Samuelson, Lifetime portfolio selection by dynamic stochastic programming, Rev. Econom. Statist., 51 (1969), pp. 239-246.

[23] S. E. Shreve And G. L. Xu, A duality method for optimal consumption and investment under shortselling prohibition: I. General market coefficients, Ann. Appl. Probab., 2 (1992), pp. 87-112.

[24] S. E. Shreve And G. L. XU, A duality method for optimal consumption and investment under shortselling prohibition: II. Constant market coefficients, Ann. Appl. Probab., 2 (1992), pp. 314-328.

[25] M. Steffensen, On the Theory of Continuous-Time Recursive Utility, working paper, 2011.

[26] R. H. Strotz, Myopia and inconsistency in dynamic utility maximization, Rev. Econom. Stud., 23 (1955), pp. 165-180.

[27] L. TEPLÁ, Optimal portfolio polices with borrowing and short sale constraints, J. Econom. Dynam. Control, 24 (2000), pp. 1623-1639.

[28] J. Wei, K. C. Wong, S. C. P. YAM, And S. P. Yung, Markowitz's mean-variance asset-liability management with regime switching: A time-consistent approach, Insurance Math. Econom., 53 (2013), pp. 281-291.

[29] K. C. Wong, Mean Variance Portfolio Selection: Time Consistent Approach, M.Phil. thesis, The University of Hong Kong, Hong Kong, 2013.

Copyright $\odot$ by SIAM. Unauthorized reproduction of this article is prohibited. 